\title{
Exact Master Equation and Quantum Decoherence of Two Coupled Harmonic Oscillators in a General Environment
}

\author{
Chung-Hsien $\mathrm{Chou}^{1,2 *}$, Ting $\mathrm{Yu}^{3 \dagger}$ and B. L. $\mathrm{Hu}^{2 \ddagger}$ \\ ${ }^{1}$ Center for Gravitation, Cosmology and Quantum Physics, \\ Institute of Physics, Academia Sinica, Nankang, Taipei 11529, Taiwan \\ ${ }^{2}$ Joint Quantum Institute and Maryland Center for Fundamental Physics, \\ Department of Physics, University of Maryland, College Park, Maryland 20742-4111 \\ ${ }^{3}$ Department of Physics and Astronomy, University of Rochester, Rochester, New York 14627-0171
}

(Dated: Second Version Dec. 2, 2007)

In this paper we derive an exact master equation for two coupled quantum harmonic oscillators interacting via bilinear coupling with a common environment at arbitrary temperature made up of many harmonic oscillators with a general spectral density function. We first show a simple derivation based on the observation that the two-harmonic oscillator model can be effectively mapped into that of a single harmonic oscillator in a general environment plus a free harmonic oscillator. Since the exact one harmonic oscillator master equation is available [Hu, Paz and Zhang, Phys. Rev. D 45, 2843 (1992)], the exact master equation with all its coefficients for this two harmonic oscillator model can be easily deduced from the known results of the single harmonic oscillator case. In the second part we give an influence functional treatment of this model and provide explicit expressions for the evolutionary operator of the reduced density matrix which are useful for the study of decoherence and disentanglement issues. We show three applications of this master equation: on the decoherence and disentanglement of two harmonic oscillators due to their interaction with a common environment under Markovian approximation, and a derivation of the uncertainty principle at finite temperature for a composite object, modeled by two interacting harmonic oscillators. The exact master equation for two, and its generalization to $N$, harmonic oscillators interacting with a general environment are expected to be useful for the analysis of quantum coherence, entanglement, fluctuations and dissipation of mesoscopic objects towards the construction of a theoretical framework for macroscopic quantum phenomena.

PACS numbers: 03.65.Yz, 03.65.Ud, 03.67.-a, 02.50.Ey

\footnotetext{
* Email Address: chouch@phys.sinica.edu.tw

† Email address: ting@pas.rochester.edu

¥ Email address: blhu@umd.edu
} 


\section{INTRODUCTION}

Macroscopic quantum coherence phenomena (MQP) manifested in double slit experiments, micromechanical resonators, Bose-Einstein condensates, Josephson junction circuits, mesoscopic systems, or even mirrors (see, e.g., [1, 2, 3, 4, 5, ㄸ, 7, 8, ㅁ, 10, 11, 12, 13, 14, 15]) is a subject of both basic theoretical and practical application interest. Theoretically it focuses on issues at the intersection of two trunk lines of important inquires in physics: the relation between the microscopic and the macroscopic world on the one hand, and the relation between the quantum and the classical on the other. Rapid recent advances in precision measurements with high degree of control and adaptability in atomic-optical, electro-mechanical, opto-mechanical, nano-material, magnetic-spin and low temperature systems have provided the rationale and substance for such theoretical investigations, and in some emergent areas where high goals are set, such as the quest for quantum information processing, even with some sense of urgency.

The issues of interest in MQP include quantum dissipation, entanglement, teleportation, decoherence, noise, correlation and fluctuations. A familiar model which one could use to address many of these issues is the quantum Brownian motion (QBM) [16, 17, 18, 19, 20] and its dynamics described by the master equation or the associated Langevin or Fokker-Planck equations. But since the systems of interest to MQP necessarily involve many microscopic or mesoscopic constituents, a many-body generalization of QBM is needed. In addition, since most of these systems involve non-negligible correlations amongst their components, quantum memory (non-Markovian) effects cannot be ignored. Even for the well-studied single harmonic oscillator (1HO) QBM, Markovian approximation is valid only for a high temperature Ohmic bath [17]. Fortunately an exact master (HPZ) equation [19] for the $1 \mathrm{HO}$ with bilinear coupling to a general environment has been found via several techniques ranging from the influence functional [19] and Wigner function [20] to quantum trajectories [21]. The $1 \mathrm{HO}$ master equation for the QBM is complex enough to encompass non-Markovian dynamics yet simple enough to yield exact solutions. (See, e.g., [22] and references therein.) The new challenge is to find the master equation for $N$ oscillators in a general environment good for the analysis of these issues in mesoscopic physics.

In this paper we show the derivation of such an equation for two coupled harmonic oscillators (2HO). A key observation is that this problem can be mapped into that of a single harmonic oscillator in a general environment plus a free harmonic oscillator. Since the master equation with all its coefficients for the 1HO QBM is known [19, 20] one can derive the master equation for the 2HO QBM easily from them. As an application of this model, we can deduce the decoherence 
properties of the $2 \mathrm{HO}$ system following the similar pattern of the $1 \mathrm{HO}$. As another example, we show explicitly how, in some parameter choice, under the Markovian limit, an entangled state evolves into a separable state in a finite time.

The results derived in this paper may be deduced by intuitive reasoning, but we are not aware of any theoretical study which yields our results. Our aim here is to provide a proof, or at least a plausibility argument, to the effect that the center of mass coordinate is the one most sensitive to the environmental influence. This model and its generalization to $N$ harmonic oscillators are expected to be useful for the analysis of quantum coherence, entanglement, fluctuations and dissipation of mesoscopic and macroscopic objects.

The paper is organized as follows: in Section II, we consider the dynamics of two harmonic oscillators coupled to a common heat bath. By employing the center of mass and relative coordinates we show how to derive the master equations of the two coupled Brownian particles. In Section III] we use the influence functional method and derive an exact form of the propagators for the reduced density matrices. These results are expected to be useful for analyzing general statistical mechanical properties of quantum open systems. In Section [V] we give three examples as applications of this master equation: the quantum decoherence and disentanglement of two interacting Brownian oscillators in a general environment, and the uncertainty relation at finite temperature for a composite object modeled by two interacting oscillators. In Section $\nabla$ we mention a few more problems and physical issues where the results from this work can be usefully applied to for their analysis and further extension of the present study. Technical details are relegated to the two appendices.

\section{THE MODEL AND THE EXACT MASTER EQUATION}

Quantum Brownian motion (QBM) of a damped harmonic oscillator bilinearly coupled to a bath of harmonic oscillators has been studied for decades, notably by Feynman-Vernon and CalderaLeggett using path integral techniques [16, 17]. For such a model an exact master equation can be deduced without making the Markovian approximation [19]. The purpose of this section is to extend the well-known Brownian motion model into the case where the system of interest contains two coupled harmonic oscillators. 


\section{A. The Model}

The Hamiltonian of the total system consisting of a system (sys) of two mutually coupled harmonic oscillators of equal mass $M$ and frequency $\Omega$ interacting with a bath (bath) of $N_{B}$ harmonic oscillators of masses $m_{n}$ and frequencies $\omega_{n}$ in an equilibrium state at a finite temperature $T$ can be formally written as,

$$
H_{\text {tot }}=H_{\text {sys }}+H_{\text {bath }}+H_{\text {int }},
$$

where

$$
H_{\mathrm{sys}}=\frac{P_{1}^{2}}{2 M}+\frac{1}{2} M \Omega^{2} x_{1}^{2}+\frac{P_{2}^{2}}{2 M}+\frac{1}{2} M \Omega^{2} x_{2}^{2}+\kappa\left(x_{1}-x_{2}\right)^{k}
$$

is the system Hamiltonian for the two system oscillators of interest, with $\left(x_{1}, x_{2}\right)$ displacements, conjugate momenta $\left(P_{1}, P_{2}\right)$ and coupling constant $\kappa$,

$$
H_{\text {bath }}=\sum_{n=1}^{N_{B}}\left(\frac{p_{n}^{2}}{2 m_{n}}+\frac{1}{2} m_{n} \omega_{n}^{2} q_{n}^{2}\right)
$$

is the bath Hamiltonian with displacement $q_{n}$ for the $n^{\text {th }}$ oscillator and conjugate momentum $p_{n}$ and

$$
H_{\mathrm{int}}=\left(x_{1}+x_{2}\right) \sum_{n=1}^{N_{B}} C_{n} q_{n}
$$

is the interaction Hamiltonian between the system and the bath. Here for simplicity, we have assumed that the two harmonic oscillators are coupled with the same coupling constants $C_{n}$ to the bath oscillators.

Our primary focus in this paper is to derive an exact master equation for the two coupled harmonic oscillators. Since the two harmonic oscillators interact with a common thermal bath, there will be induced coupling between the two harmonic oscillators even when initially they are uncoupled. Thus, the master equation for $2 \mathrm{HO} \mathrm{QBM}$ is not simply the addition of the two master equations for 1HO QBM. It must account for the mutual interactions between the two Brownian particles introduced by their coupling to the common heat bath. Of interest is a comparison with the model that consists of $2 \mathrm{HO}$ each in its own heat bath. In our model, the coupling to a common heat bath can give rise to several new features, of particular interest here is the generation of entanglement between the two Brownian particles due to the back-action of the heat bath on the system [24, 25, 26, 27]. 
However, as is well-known for classical mechanics, the dynamics of an $\mathrm{N}$ body quantum open system can be made simpler by changing the $\mathrm{N}$ body coordinates to that of their center of mass $(\mathrm{cm})$ and relative (rel) coordinates. Here, the difference is that the $\mathrm{N}$ harmonic oscillators (NHO) are coupled with an environment and we seek a quantum mechanical treatment. A quantum mechanical theory of $\mathrm{N}$ body dynamics forms the theoretical basis for treating MQP. In this paper we treat the $2 \mathrm{HO}$ case. We will show in what follows that the exact master equation for the two coupled harmonic oscillators can be obtained directly from the master equation for the single harmonic oscillator, known as the Hu-Paz-Zhang (HPZ) master equation.

Let us first rewrite the total Hamiltonian in terms of a set of new variables $X, x, P, p$ defined as

$$
\begin{aligned}
& X=\frac{1}{2}\left(x_{1}+x_{2}\right), \quad x=x_{1}-x_{2}, \\
& P=P_{1}+P_{2}, \quad p=\frac{1}{2}\left(P_{1}-P_{2}\right),
\end{aligned}
$$

and the new masses $M_{1}=2 M, M_{2}=M / 2$. In terms of these new variables the Hamiltonian (1) takes the following form:

$$
H_{\mathrm{sys}}=H_{\mathrm{cm}}+H_{\mathrm{rel}}
$$

where

$$
\begin{gathered}
H_{\mathrm{cm}}=\frac{P^{2}}{2 M_{1}}+\frac{1}{2} M_{1} \Omega^{2} X^{2}, \\
H_{\text {rel }}=\frac{p^{2}}{2 M_{2}}+\frac{1}{2} M_{2} \Omega^{2} x^{2}+\kappa x^{k},
\end{gathered}
$$

and

$$
H_{\mathrm{int}}=\left(x_{1}+x_{2}\right) \sum_{n=1}^{N_{B}} C_{n} q_{n}=2 X \sum_{n=1}^{N_{B}} C_{n} q_{n}=X \sum_{n=1}^{N_{B}} \tilde{C}_{n} q_{n}
$$

where $\tilde{C}_{n}=2 C_{n}$ are modified coupling constants. Since (5) and (6) are canonical transformations, all the commutators are preserved, and it is easy to check that

$$
[X, P]=[x, p]=i \hbar, \quad[P, x]=[p, X]=[X, x]=[P, p]=0 .
$$

We see that the fictitious particle with mass $M_{2}$ and dynamical variables $x, p$ has no interaction with either the cm particle with mass $M_{1}$ with canonical variables $X, P$ or the oscillators of the heat bath with canonical variables $q_{n}$. 
The total Hamiltonian $H_{\text {tot }}$ in (1) can now be written as $H_{\text {tot }}=H_{\text {tot }}^{\prime}+H_{\text {rel }}$ with a new effective total Hamiltonian

$$
\begin{aligned}
H_{\mathrm{tot}}^{\prime}= & H_{\mathrm{cm}}+H_{\mathrm{int}}+H_{\mathrm{bath}} \\
& =\frac{P^{2}}{2 M_{1}}+\frac{1}{2} M_{1} \Omega^{2} X^{2}+X \sum_{n=1}^{N_{B}} \tilde{C}_{n} q_{n}+\sum_{n=1}^{N_{B}}\left(\frac{p_{n}^{2}}{2 m_{n}}+\frac{1}{2} m_{n} \Omega_{n}^{2} q_{n}^{2}\right) .
\end{aligned}
$$

This Hamiltonian is formally the same as the Hamiltonian for the single harmonic oscillator in $\mathrm{cm}$ variables $(X, P)$ coupled to the heat bath with coupling constants $\tilde{C}_{n}$. Note that for this case the spectral density $\tilde{I}(\omega)$ is given by:

$$
\tilde{I}(\omega)=\pi \sum_{n=1}^{N_{B}} \frac{{\tilde{C_{n}}}^{2}}{2 m_{n} \omega_{n}} \delta\left(\omega-\omega_{n}\right),
$$

which differs from the original spectral density $I(\omega)$ by a numerical factor 4 .

\section{B. Density Matrix}

We now consider the dynamics of two coupled harmonic oscillators interacting with a common heat bath. The density matrix $\rho$ evolves in time under the unitary operator:

$$
\rho(t)=\exp \left[-i \frac{H_{\mathrm{tot}} t}{\hbar}\right] \rho(0) \exp \left[i \frac{H_{\mathrm{tot}} t}{\hbar}\right] .
$$

From (12), it is easy to see that this evolution can be decomposed into two parts, a dissipative evolution of the center of mass system,

$$
\tilde{\rho}(t)=\exp \left[-i \frac{H_{\mathrm{tot}}^{\prime} t}{\hbar}\right] \rho(0) \exp \left[i \frac{H_{\mathrm{tot}}^{\prime} t}{\hbar}\right],
$$

and the unitary evolution of the free harmonic oscillator with mass $M_{1}$,

$$
\rho(t)=\exp \left[-i \frac{H_{\mathrm{rel}} t}{\hbar}\right] \tilde{\rho}(t) \exp \left[i \frac{H_{\mathrm{rel}} t}{\hbar}\right],
$$

where $H_{\text {rel }}$ is the Hamiltonian for the $1 \mathrm{HO}$ system with reduced mass $M_{2}=M / 2$ and $x, p$ variables:

$$
H_{\text {rel }}=\frac{p^{2}}{2 M_{2}}+\frac{1}{2} M_{2} \Omega^{2} x^{2}+\kappa x^{k} .
$$

For technical simplicity we make the usual assumption that the initial state of the total system is uncorrelated,

$$
\rho(0)=\rho_{\text {sys }}(0) \times \rho_{\text {bath }}(0),
$$

and that the heat bath is in a thermal equilibrium state at temperature $T$. 


\section{Exact Master Equation}

If we are interested in the detailed dynamics of the system but only the coarse-grained effect of the bath we can work with the reduced density matrix obtained by tracing $\rho$, the density matrix of the total system described by (11), over the bath variables [28, 29]:

$$
\rho_{r}=\operatorname{Tr}_{\text {bath }} \rho(t)
$$

The reduced density operator for the center of mass system is obtained in a similar way,

$$
\tilde{\rho}_{r}=\operatorname{Tr}_{\text {bath }} \tilde{\rho}(t)
$$

where $\tilde{\rho}$ defined in (15) is the density operator for the effective total system (12). The relationship between $\tilde{\rho}_{r}$ and $\rho_{r}$ is given by

$$
\rho_{r}(t)=\exp \left[-i \frac{H_{\mathrm{rel}} t}{\hbar}\right] \tilde{\rho}_{r}(t) \exp \left[i \frac{H_{\mathrm{rel}} t}{\hbar}\right] .
$$

Tracing over the heat bath variables in (15) leads us to a HPZ type master equation for the center of mass variables $X, P$ :

$$
\dot{\tilde{\rho}}_{r}=\frac{1}{i \hbar}\left[H_{\mathrm{cm}}, \tilde{\rho}_{r}\right]+\frac{a(t)}{2 i \hbar}\left[X^{2}, \tilde{\rho}_{r}\right]+\frac{b(t)}{2 i \hbar}\left[X,\left\{P, \tilde{\rho}_{r}\right\}\right]+\frac{c(t)}{\hbar^{2}}\left[X,\left[P, \tilde{\rho}_{r}\right]\right]-\frac{d(t)}{\hbar^{2}}\left[X,\left[X, \tilde{\rho}_{r}\right]\right]
$$

Note here that $H_{\mathrm{cm}}$ defined in (9) is the Hamiltonian for the center of mass variables $X, P$ only. This is the exact master equation for $X, P$ interacting with a thermal heat bath with the spectral density $\tilde{I}(\omega)$ rather than $I(\omega)$. As a consequence, the coefficients $a, b, c, d$ in the above master equation satisfy the same types of equations given by [19] (or [20]), only the coupling constants and mass are different here.

From the evolution equation (16), the required master equation for the reduced density matrix $\rho_{r}(t)$ is thus obtained,

$$
\dot{\rho}_{r}=\frac{1}{i \hbar}\left[H_{\mathrm{sys}}, \rho_{r}\right]+\frac{a(t)}{2 i \hbar}\left[X^{2}, \rho_{r}\right]+\frac{b(t)}{2 i \hbar}\left[X,\left\{P, \rho_{r}\right\}\right]+\frac{c(t)}{\hbar^{2}}\left[X,\left[P, \rho_{r}\right]\right]-\frac{d(t)}{\hbar^{2}}\left[X,\left[X, \rho_{r}\right]\right] .
$$

The only difference between Eq. (23) and Eq. (22) is that the unitary evolution is modified by the fictitious harmonic oscillator $x, p$.

In terms of the original variables $x_{1}, x_{2}, P_{1}, P_{2}$, we get

$$
\begin{aligned}
\dot{\rho}_{r}= & \frac{1}{i \hbar}\left[H_{\mathrm{sys}}, \rho_{r}\right]+\frac{a(t)}{8 i \hbar}\left[\left(x_{1}+x_{2}\right)^{2}, \rho_{r}\right]+\frac{b(t)}{4 i \hbar}\left[x_{1}+x_{2},\left\{P_{1}+P_{2}, \rho_{r}\right\}\right] \\
& +\frac{c(t)}{2 \hbar^{2}}\left[x_{1}+x_{2},\left[P_{1}+P_{2}, \rho_{r}\right]\right]-\frac{d(t)}{4 \hbar^{2}}\left[x_{1}+x_{2},\left[x_{1}+x_{2}, \rho_{r}\right]\right] .
\end{aligned}
$$


This is the exact master equation for the two coupled harmonic oscillators. In the coordinate representation,

$$
\rho_{r}\left(x_{1}, x_{2}, y_{1}, y_{2}\right) \equiv\left\langle x_{1}, x_{2}\left|\rho_{r}\right| y_{1}, y_{2}\right\rangle
$$

the master equation can be easily written as:

$$
\begin{aligned}
i \hbar \frac{\partial \rho_{r}}{\partial t}= & -\frac{\hbar^{2}}{2 M}\left(\frac{\partial^{2}}{\partial x_{1}^{2}}-\frac{\partial^{2}}{\partial y_{1}^{2}}+\frac{\partial^{2}}{\partial x_{2}^{2}}-\frac{\partial^{2}}{\partial y_{2}^{2}}\right) \rho_{r}+\frac{1}{2} M \Omega^{2}\left(x_{1}^{2}-y_{1}^{2}+x_{2}^{2}-y_{2}^{2}\right) \rho_{r} \\
& +\frac{1}{2} M \delta \Omega^{2}(t)\left(x_{1}-y_{1}+x_{2}-y_{2}\right) \frac{1}{2}\left(x_{1}+y_{1}+x_{2}+y_{2}\right) \rho_{r} \\
& -i \hbar \Gamma(t)\left(x_{1}-y_{1}+x_{2}-y_{2}\right) \frac{1}{2}\left(\frac{\partial}{\partial x_{1}}-\frac{\partial}{\partial y_{1}}+\frac{\partial}{\partial x_{2}}-\frac{\partial}{\partial y_{2}}\right) \rho_{r} \\
& -i M \Sigma(t)\left(x_{1}-y_{1}+x_{2}-y_{2}\right)^{2} \rho_{r} \\
& +\hbar \Delta(t)\left(x_{1}-y_{1}+x_{2}-y_{2}\right)\left(\frac{\partial}{\partial x_{1}}+\frac{\partial}{\partial y_{1}}+\frac{\partial}{\partial x_{2}}+\frac{\partial}{\partial y_{2}}\right) \rho_{r} .
\end{aligned}
$$

A set of new notations in (26) is introduced to facilitate easy adoption of results from [19]. In particular,

$$
\begin{aligned}
& a(t)=M \delta \Omega^{2}(t), \quad b(t)=2 \Gamma(t), \\
& c(t)=\Delta(t), \quad d(t)=\Sigma(t) .
\end{aligned}
$$

It is often useful to use the Wigner function defined in phase space, which is related to the reduced density matrix $\rho_{r}$ in the following way:

$$
\begin{aligned}
\tilde{W}\left(x_{1}, x_{2}, P_{1}, P_{2}, t\right)= & \frac{1}{(2 \pi)^{2}} \int d u_{1} d u_{2} e^{i\left(u_{1} P_{1}+u_{2} P_{2}\right) / \hbar} \\
& \times \rho_{r}\left(x_{1}-\frac{u_{1}}{2}, x_{2}-\frac{u_{2}}{2} ; x_{1}+\frac{u_{1}}{2}, x_{2}+\frac{u_{2}}{2}, t\right) .
\end{aligned}
$$

In correspondence with (26) the Wigner function satisfies a Fokker-Planck equation:

$$
\begin{aligned}
\frac{\partial \tilde{W}}{\partial t}= & -\sum_{i=1,2}\left(\frac{P_{i}}{M} \frac{\partial \tilde{W}}{\partial x_{i}}-M \Omega^{2} x_{i} \frac{\partial \tilde{W}}{\partial P_{i}}\right) \\
& +M \delta \Omega^{2}(t)\left(x_{1}+x_{2}\right)\left(\frac{\partial}{\partial P_{1}}+\frac{\partial}{\partial P_{2}}\right) \tilde{W}+2 \Gamma(t)\left(\frac{\partial}{\partial P_{1}}+\frac{\partial}{\partial P_{2}}\right)\left[\left(P_{1}+P_{2}\right) \tilde{W}\right] \\
& +\Sigma(t)\left(\frac{\partial}{\partial P_{1}}+\frac{\partial}{\partial P_{2}}\right)^{2} \tilde{W}+\Delta(t)\left(\frac{\partial}{\partial P_{1}}+\frac{\partial}{\partial P_{2}}\right)\left(\frac{\partial}{\partial x_{1}}+\frac{\partial}{\partial x_{2}}\right) \tilde{W} .
\end{aligned}
$$

The time-dependent functions $\delta \Omega^{2}(t), \Gamma(t), \Delta(t), \Sigma(t)$ are derived following the same method used by HPZ which can be found in Appendix A.5.

In deriving the exact master equation we assumed that the initial state for the two harmonic oscillators is a product of a function of the relative coordinates and a function of the center of mass coordinates. However, it can be easily shown that the derivation is valid for an arbitrary initial state of the system regardless of the condition of separability. 


\section{Markov Approximations}

The derived master equation (26) is exact, so it is valid in both the Markovian and the nonMarkovian regimes. Memory effects due to the environment is encoded in the time-dependent coefficients. In the high temperature ohmic bath limit, the coefficients become constants and the spectral density has the form:

$$
I(\omega)=M_{1} \gamma \omega \exp \left(-\frac{\omega^{2}}{\Lambda^{2}}\right)
$$

where $\Lambda$ is a cut-off frequency. In the so-called Fokker-Planck limit $\left(k_{B} T \gg \hbar \Lambda\right)$, we have

$$
\nu(s)=\frac{2 M_{1} k_{B} T \gamma}{\hbar} \delta(s), \eta(s)=M \gamma \frac{d}{d s} \delta(s) .
$$

Hence, $\delta \Omega^{2}=-2 \gamma \delta(0), \Gamma=\gamma, \Delta=0, \Sigma=2 M_{1} \gamma k_{B} T$. The constant coefficients obtained for such a model give rise to a Markovian master equation. The Wigner function for the center of mass coordinates obeys the Fokker-Planck-Markov equation [30]:

$$
\begin{aligned}
\frac{\partial W_{\mathrm{cm}}}{\partial t}= & -\frac{P}{M_{1}} \frac{\partial W_{\mathrm{cm}}}{\partial X}-M_{1} \Omega^{\prime 2} X^{2} \frac{\partial W_{\mathrm{cm}}}{\partial P} \\
& +2 \gamma \frac{\partial}{\partial P}\left(P W_{\mathrm{cm}}\right) \\
& +2 M_{1} \gamma k_{B} T \frac{\partial^{2}}{\partial P^{2}} W_{\mathrm{cm}},
\end{aligned}
$$

where $M_{1}=2 M$ and $\Omega^{\prime 2}=\Omega^{2}+\delta \Omega^{2}$.

\section{THE INFLUENCE FUNCTIONAL METHOD}

In the last section we showed a simple derivation of the master equation for the reduced density matrix and the Fokker-Planck equation for the Wigner function. In general it is difficult to get a general analytical solution of the master equation. It turns out that in some cases of interest, one can get analytic solutions of the master equation through the influence functional method [31]. Using this method, we can get the evolution operator for the reduced density matrix or the evolution kernel for the exact master equation which will be very useful for the study of quantum decoherence and disentanglement problems.

Because of this, in this subsection, we will outline the key steps in the derivation of the master equation (26) via the path integral method.

As before, the density matrix of the total system at any time $t$ can be written as

$$
\rho(t)=e^{-i \frac{H_{\mathrm{tot} t}}{\hbar}} \rho(0) e^{i \frac{H_{\mathrm{tot} t}}{\hbar}} .
$$


The reduced density matrix of the system is evolved by the propagator $J_{r}$ from time $t=0$ to $t$ as

$$
\begin{aligned}
\rho_{r}\left(x_{1}, x_{2} ; y_{1}, y_{2}, t\right)= & \int d q_{n}\left\langle x_{1}, x_{2}, q_{n}|\rho(t)| y_{1}, y_{2}, q_{n}\right\rangle \\
= & \int d x_{0} d y_{0} J_{r}\left(x_{1}, x_{2}, y_{1}, y_{2}, t ; x_{10}, x_{20}, y_{10}, y_{20}, 0\right) \\
& \times \rho_{r}\left(x_{10}, x_{20} ; y_{10}, y_{20} ; t=0\right),
\end{aligned}
$$

where we have used the collective notation $d x_{0} d y_{0}=d x_{10} d x_{20} d y_{10} d y_{20}$.

The evolution propagator $J_{r}$ can be written in a path-integral representation as

$$
\begin{aligned}
& J_{r}\left(x_{1}, x_{2}, y_{1}, y_{2}, t ; x_{1}^{\prime}, x_{2}^{\prime}, y_{1}^{\prime}, y_{2}^{\prime}, 0\right) \\
= & \prod_{k=1}^{2} \int_{x_{k i}}^{x_{k f}} \mathcal{D} x_{k} \int_{y_{k i}}^{y_{k f}} \mathcal{D} y_{k} \exp \left(\frac{i}{\hbar} S_{S}\left[x_{1}, x_{2}\right]-\frac{i}{\hbar} S_{S}\left[y_{1}, y_{2}\right]\right) \times \mathcal{F}\left[x_{1}, x_{2}, y_{1}, y_{2}\right],
\end{aligned}
$$

where $\mathcal{F}\left[x_{1}, x_{2}, y_{1}, y_{2}\right]$ is the Feynman-Vernon influence functional defined by

$$
\begin{aligned}
\mathcal{F}\left[x_{1}, x_{2}, y_{1}, y_{2}\right]= & \int d q_{n}^{\prime} d \tilde{q}_{n}^{\prime} d q_{n} \rho_{b a t h}\left(q_{n}^{\prime}, \tilde{q}_{n}^{\prime}, 0\right) \int_{q_{n}^{\prime}}^{q_{n}} \mathcal{D} q_{n} \int_{\tilde{q}_{n}^{\prime}}^{q_{n}} \mathcal{D} \tilde{q}_{n} \exp \left\{\frac { i } { \hbar } \left(S_{I}\left[x_{1}, x_{2}, q_{n}\right]-\right.\right. \\
& \left.\left.S_{I}\left[y_{1}, y_{2}, \tilde{q}_{n}\right]+S_{B}\left[q_{n}\right]-S_{B}\left[\tilde{q}_{n}\right]\right)\right\} \\
= & \exp \left\{\frac{i}{\hbar}\left(S_{I F}\left[x_{1}, x_{2}, y_{1}, y_{2}\right]\right)\right\},
\end{aligned}
$$

where $S_{I F}$ is the influence action. For the QBM model we are considering here, the influence action can be written as:

$$
\begin{aligned}
S_{I F}\left[x_{1}, x_{2}, y_{1}, y_{2}\right] & =-2 \int_{0}^{t} d s_{1} \int_{0}^{s_{1}} d s_{2}\left[\Delta_{1}\left(s_{1}\right)+\Delta_{2}\left(s_{1}\right)\right] \eta\left(s_{1}-s_{2}\right)\left[\Sigma_{1}\left(s_{2}\right)+\Sigma_{2}\left(s_{2}\right)\right] \\
& +i \int_{0}^{t} d s_{1} \int_{0}^{s_{1}} d s_{2}\left[\Delta_{1}\left(s_{1}\right)+\Delta_{2}\left(s_{1}\right)\right] \nu\left(s_{1}-s_{2}\right)\left[\Delta_{1}\left(s_{2}\right)+\Delta_{2}\left(s_{2}\right)\right]
\end{aligned}
$$

where

$$
\Sigma_{1}=\frac{1}{2}\left(x_{1}+y_{1}\right), \quad \Sigma_{2}=\frac{1}{2}\left(x_{2}+y_{2}\right), \quad \Delta_{1}=x_{1}-y_{1}, \quad \Delta_{2}=x_{2}-y_{2} .
$$

Note that the integrand in Eq. (36) is Gaussian, hence the integral can be computed exactly and the explicit form of $J_{r}$ is,

$$
J_{r}=\tilde{N} \exp \left(\frac{i}{2} S_{I}-S_{R}\right)
$$

where the expressions of $S_{I}$ and $S_{R}$ can be written in more compact forms with the following notations:

$$
\begin{aligned}
& x_{k}^{+}=x_{1 k}+x_{2 k}, \quad y_{k}^{+}=y_{1 k}+y_{2 k}, \\
& x_{k}^{-}=x_{1 k}-x_{2 k}, \quad y_{k}^{-}=y_{1 k}-y_{2 k},
\end{aligned}
$$


whence

$$
\begin{aligned}
S_{I} & =b_{1}\left(x_{t}^{+}+y_{t}^{+}\right)\left(x_{t}^{+}-y_{t}^{+}\right)+b_{2}\left(x_{0}^{+}+y_{0}^{+}\right)\left(x_{t}^{+}-y_{t}^{+}\right) \\
& -b_{3}\left(x_{t}^{+}+y_{t}^{+}\right)\left(x_{0}^{+}-y_{0}^{+}\right)-b_{4}\left(x_{0}^{+}+y_{0}^{+}\right)\left(x_{0}^{+}-y_{0}^{+}\right) \\
& +b_{5}\left(x_{t}^{-}+y_{t}^{-}\right)\left(x_{t}^{-}-y_{t}^{-}\right)+b_{6}\left(x_{0}^{-}+y_{0}^{-}\right)\left(x_{t}^{-}-y_{t}^{-}\right) \\
& -b_{7}\left(x_{t}^{-}+y_{t}^{-}\right)\left(x_{0}^{-}-y_{0}^{-}\right)-b_{8}\left(x_{0}^{-}+y_{0}^{-}\right)\left(x_{0}^{-}-y_{0}^{-}\right),
\end{aligned}
$$

and

$$
\begin{aligned}
S_{R}= & a_{11}\left(x_{t}^{+}-y_{t}^{+}\right)^{2}+a_{22}\left(x_{0}^{+}-y_{0}^{+}\right)^{2} \\
& +a_{12}\left(x_{0}^{+}-y_{0}^{+}\right)\left(x_{t}^{+}-y_{t}^{+}\right) .
\end{aligned}
$$

The functions $b_{i}(t)$ and $a_{i j}(t)$ depend on the environment and can be constructed from the solutions to the equations

$$
\begin{aligned}
& b_{2}(t) \equiv \frac{1}{2} \dot{u}_{1}(t), \quad b_{1}(t) \equiv \frac{1}{2} \dot{u}_{2}(t), \quad b_{6}(t) \equiv \frac{1}{2} \dot{w}_{1}(t), \quad b_{5}(t) \equiv \frac{1}{2} \dot{w}_{2}(t), \\
& b_{4}(t) \equiv \frac{1}{2} \dot{u}_{1}(0), \quad b_{3}(t) \equiv \frac{1}{2} \dot{u}_{2}(0), \quad b_{8}(t) \equiv \frac{1}{2} \dot{w}_{1}(0), \quad b_{7}(t) \equiv \frac{1}{2} \dot{w}_{2}(0),
\end{aligned}
$$

where $w_{i}(t)$ are functions which satisfy the following equation

$$
\ddot{\bar{\Sigma}}(s)+\Omega^{2} \bar{\Sigma}(s)=0,
$$

with the boundary conditions:

$$
\begin{gathered}
w_{1}(0)=1=w_{2}(t), \quad w_{1}(t)=0=w_{2}(0), \\
a_{i j}(t)=\frac{1}{2} \int_{0}^{t} d s_{1} \int_{0}^{t} d s_{2} u_{i}\left(s_{1}\right) \nu\left(s_{1}-s_{2}\right) u_{j}\left(s_{2}\right) .
\end{gathered}
$$

With the expression of $J_{r}$, we can derive the master equation for the reduced density matrix (26). This is shown in Appendix A.

An exact form of the evolutionary operator for the reduced density matrix is a priced object: Not only can one derive from it the exact master equation for the reduced density matrix, with this explicit expression of the evolutionary operator, given any initial reduced density matrix $\rho_{r}$ at time $t_{0}$ one can calculate $\rho_{r}$ at any later time $t$ without having to solve the complicated second order partial differential equation with time-dependent coefficient functions.

For example, we will apply this evolutionary operator to the study of the decoherence and disentanglement of two coupled harmonic oscillators in a common heat bath. One can also use 
it to calculate the higher moments of physical observables of interest such as the position and the momentum operators which enter into the derivation of a generalized uncertainty principle for composite objects at finite temperature [32]. It can also be used to address the issue of the influence of entanglement on the relation between the statistical entropy of an open quantum system and the heat exchanged with a low temperature environment such as studied in [50]. Another interesting application would be the entanglement between a qubit and an oscillator. Adopting a level reduction scheme, Shiokawa and $\mathrm{Hu}$ [41] used the evolutionary operator of 1HO QBM to study the dynamics of the spin-boson model. The explicit expression of the evolutionary operator for the $2 \mathrm{HO} \mathrm{QBM}$ may be used to construct effective 1HO-spin-boson models found in many condensed matter quantum computer schemes for the analysis of the interaction between a qubit and a harmonic oscillator and their decoherence and disentanglement dynamics in the presence of a general environment. See Section $\mathrm{V}$ for a more detailed exposition of further applications and extensions.

\section{APPLICATIONS: QUANTUM DECOHERENCE AND DISENTANGLEMENT, UNCERTAINTY RELATION FOR A COMPOSITE OBJECT}

In this section we give three examples for the application of this master equation: the decoherence and disentanglement of two coupled harmonic oscillators in a common heat bath, and a derivation of the uncertainty relation at finite temperature for a composite object modeled by two harmonic oscillators in a general environment. For some simplified cases we obtain analytic results which show interesting features such as finite-time disentanglement [33, 34].

\section{A. Dynamics of Quantum Coherence}

We will assume that the system and the environment are initially uncorrelated. The total density matrix at time $t=0$ then factorizes into a product of density matrices for the system and the environment. As usual, we further assume that the environment is initially in thermal equilibrium at a given temperature $T$.

We assume initially the $2 \mathrm{HO}$ (labeled as 1 and 2) are separated with distance $2 L_{0}$ and the initial wave function of the 1-2 system is given by

$$
\begin{aligned}
\Psi\left(x_{1}, x_{2}, t=0\right)= & s_{1} \Psi_{1}\left(x_{1}\right) \Psi_{1}\left(x_{2}\right)+s_{2} \Psi_{1}\left(x_{1}\right) \Psi_{2}\left(x_{2}\right) \\
& +s_{3} \Psi_{2}\left(x_{1}\right) \Psi_{1}\left(x_{2}\right)+s_{4} \Psi_{2}\left(x_{1}\right) \Psi_{2}\left(x_{2}\right),
\end{aligned}
$$


where we have defined the displaced Gaussian states as

$$
\Psi_{1,2}(x)=N \exp \left[-\frac{\left(x \mp L_{0}\right)^{2}}{2 \delta^{2}}\right] \exp \left( \pm i P_{0} x\right)
$$

and $s_{i}$ are any complex numbers subject to normalization conditions. (We use 1,2 to label different initial positions of the center of the Gaussian wave function of harmonic oscillators while $x, y$ label different time paths.)

With an initial reduced density matrix

$$
\begin{aligned}
\rho_{r}\left(x_{10}, x_{20} ; y_{10}, y_{20} ; t=0\right) & =\left\langle x_{10}, x_{20} \mid \Psi(0)\right\rangle\left\langle\Psi(0) \mid y_{10}, y_{20}\right\rangle \\
& \equiv \sum_{i, j} s_{i} s_{j}^{*} \rho_{i j}\left(x_{10}, x_{20} ; y_{10}, y_{20} ; t=0\right),
\end{aligned}
$$

the reduced density matrix at $t$ is given by

$$
\begin{aligned}
\rho_{r}\left(x_{1}, x_{2} ; y_{1}, y_{2} ; t\right)= & \int d x_{0} d y_{0} J_{r}\left(x_{1}, x_{2}, y_{1}, y_{2}, t ; x_{10}, x_{20}, y_{10}, y_{20}, 0\right) \\
& \times \rho_{r}\left(x_{10}, x_{20} ; y_{10}, y_{20} ; t=0\right) .
\end{aligned}
$$

Because the QBM model is linear and the initial state is Gaussian, we can solve the master equation exactly for the dynamics of the $2 \mathrm{HO}$ system interacting with an environment with a general spectral density at any temperature. Therefore, we can obtain the total density matrix if the explicit solutions for each component are known,

$$
\begin{aligned}
\rho_{i j}\left(x_{1}, x_{2} ; y_{1}, y_{2} ; t\right)= & \int d x_{0} d y_{0} J_{r}\left(x_{1}, x_{2}, y_{1}, y_{2}, t ; x_{10}, x_{20}, y_{10}, y_{20}, 0\right) \\
& \times \rho_{i j}\left(x_{10}, x_{20} ; y_{10}, y_{20} ; t=0\right) .
\end{aligned}
$$

Note that since $J_{r}$ and $\rho_{i j}$ are in the form of an exponential with an exponent which is a quadratic function in $\left(x_{10}, x_{20} ; y_{10}, y_{20}\right)$, we can use a standard trick for the evaluation of the integral,

$$
\begin{aligned}
\rho_{i j}(t) & =\int d x_{0} d y_{0} J_{t} \times \rho_{i j}(t=0) \\
& =\int d x_{0} d y_{0} \exp \left[-\vec{x}^{T} \cdot G_{i j} \cdot \vec{x}+\frac{1}{2} \vec{F}_{i j}^{T} \cdot \vec{x}+\frac{1}{2} \vec{x}^{T} \cdot \vec{F}_{i j}+c_{i j}\right] \\
& =\frac{(\sqrt{\pi})^{4}}{\sqrt{\operatorname{det} G_{i j}}} \exp \left(c_{i j}+\frac{1}{4} \vec{F}_{i j}^{T} \cdot G_{i j}^{-1} \cdot \vec{F}_{i j}\right),
\end{aligned}
$$

where $\vec{x}^{T}=\left(x_{10}, x_{20}, y_{10}, y_{20}\right)$.

Once we have $\rho_{i j}\left(x_{1}, x_{2} ; y_{1}, y_{2} ; t\right)$ we can perform the following substitution $x_{1} \mapsto X_{1}-\frac{z_{1}}{2} ; x_{2} \mapsto$ $X_{2}-\frac{z_{2}}{2} ; y_{1} \mapsto X_{1}+\frac{z_{1}}{2} ; y_{2} \mapsto X_{2}+\frac{z_{2}}{2}$ and then do the Fourier transform to get the Wigner function 
at a later time $t$ :

$$
\begin{aligned}
W_{i j}\left(X_{1}, X_{2}, P_{1}, P_{2}, t\right)= & \iint \frac{d z_{1} d z_{2}}{(2 \pi \hbar)^{2}} \exp \left(i P_{1} z_{1}+i P_{2} z_{2}\right) \\
& \times \rho_{i j}\left(X_{1}-\frac{z_{1}}{2}, X_{2}-\frac{z_{2}}{2} ; X_{1}+\frac{z_{1}}{2}, X_{2}+\frac{z_{2}}{2} ; t\right) .
\end{aligned}
$$

Since after the substitution the exponent of $\rho_{i j}$ is quadratic in $z_{1}, z_{2}$, the above integration can be evaluated explicitly. These solutions (54) and (55) will be useful in decoherence and disentanglement analysis below. The detailed results and the explicit expressions of $\rho_{i j}$ can be found in Appendix B.

When viewed from the center of mass coordinate the physics of decoherence for a $2 \mathrm{HO}$ system is essentially similar to that described in [19, 35] using the Hu-Paz-Zhang master equation for 1HO because the environment couples to the system only through the center of mass coordinate $X$ and is independent of the relative coordinate $x$. The evolution of the relative coordinate part in the reduced density matrix is unitary and hence will not affect the decoherence processes. One can easily recognize these features from (22) and (21). The effects of environment-induced decoherence are encoded in the coefficient functions $a(t), b(t), c(t), d(t)$ of (22). As one can see from this example four of the matrix elements $\rho_{11}, \rho_{14}, \rho_{41}, \rho_{44}$ are similar to those in the example considered in [35] sans the relative coordinates.

However, the issue of disentanglement is quite different because usually the entanglement measure is related to the global property of the whole reduced density matrix. In general, entanglement involves both the center of mass and the relative coordinate dynamics. It is difficult to make any prediction on how disentanglement evolves from the information of only the $1 \mathrm{HO}$ system. For instance, while the cm coherence always disappear asymptotically, in contrast, entanglement of the two particles may terminate in a finite time. In the third subsection, we will address this issue with a simple illustrative example.

\section{B. Uncertainty Principle for Composite Objects}

In this subsection, the generalized uncertainty relation for a composite object is investigated from the viewpoint of quantum open systems. Here the system is modeled by two harmonic oscillators and the environment by a heat bath at temperature $T$. As such, both thermal fluctuation and quantum noise come to play when the uncertainty relation between position and momentum is considered [19, 32].

The exact solution for the two harmonic oscillators coupled to a common heat bath can be found 
by decomposing the total system into two fictitious surrogate subsystems, namely, the subsystems described by the center of mass and the relative coordinates, respectively. Such a decomposition guarantees that the two subsystems are decoupled, and as such, the solution of the total system is a tensor product of the two subsystems:

$$
\rho_{r}=\rho_{\mathrm{cm}} \otimes \rho_{\mathrm{rel}}
$$

Using the center of mass coordinate as described by the Hamiltonian (1), the complete information about the state of the open system is contained in the reduced density operator $\rho_{r}(t)$.

For a class of initial Gaussian states given by

$$
\psi(x, 0)=N_{0} \exp \left[-\frac{\left(x-x_{0}\right)^{2}}{4 \sigma^{2}}+\frac{i}{\hbar} p_{0} x\right]
$$

where, $N_{0}=1 /\left(2 \pi \sigma^{2}\right)^{\frac{1}{4}}$, the initial density operator for each fictitious harmonic oscillator in the coordinate representation can be written as:

$$
\rho\left(x, x^{\prime}, 0\right)=\psi^{*}(x, 0) \psi\left(x^{\prime}, 0\right)=N_{0}^{2} \exp \left[-\frac{\left(x-x_{0}\right)^{2}}{4 \sigma^{2}}-\frac{\left(x^{\prime}-x_{0}\right)^{2}}{4 \sigma^{2}}-\frac{i}{\hbar} p_{0} x+\frac{i}{\hbar} p_{0} x^{\prime}\right] .
$$

In order to compute the variance of position and momentum operators, it is more convenient to use the Wigner function which can be obtained from the Fourier transform of the density operators (29). To be more specific, for the harmonic oscillator representing the center of mass degree of freedom, the corresponding Wigner function is simply given by:

$$
W_{\mathrm{cm}}(X, P)=N_{0}^{2} \exp \left[-\frac{\left(X-x_{0}\right)^{2}}{2 \sigma^{2}}-\frac{2 \sigma^{2}}{\hbar^{2}}\left(P-p_{0}\right)^{2}\right]
$$

The variance of the operator $X, P$ denoted by $(\Delta X)^{2}=\left\langle X^{2}\right\rangle-\langle X\rangle^{2}$ and $(\Delta P)^{2}=\left\langle P^{2}\right\rangle-\langle P\rangle^{2}$ can be computed easily

$$
\begin{aligned}
\left\langle X^{2}\right\rangle & =\frac{1}{2 \pi \hbar} \int d X d P X^{2} W_{\mathrm{cm}}(X, P, t) \\
\left\langle P^{2}\right\rangle & =\frac{1}{2 \pi \hbar} \int d X d P P^{2} W_{\mathrm{cm}}(X, P, t)
\end{aligned}
$$

where $W_{\mathrm{cm}}(X, P, t)$ is the solution of the Fokker-Planck equation for a single harmonic oscillator (see Appendix B or [32]). In particular, for an ohmic environment (32), the uncertainly relation in the weak damping limit $(\gamma<<2 \Omega)$ is given by

$$
U(t)=(\Delta X)^{2}(\Delta P)^{2}(\Delta x)^{2}(\Delta p)^{2}=f_{\mathrm{cm}}(t) f_{\mathrm{rel}}(t)
$$


with

$$
\begin{aligned}
f_{\mathrm{cm}}(t) & =\frac{\hbar^{2}}{4}\left[e^{-\gamma t}+\operatorname{coth} \frac{\hbar \Omega^{\prime}}{2 k_{B} T}\left(1-e^{-\gamma t}\right)\right]^{2} \\
& +\hbar^{2} \operatorname{coth} \frac{\hbar \Omega^{\prime}}{2 k_{B} T}\left[\frac{(1-\delta)^{2}}{4 \delta}\left(1-e^{-\gamma t}\right)-\frac{\left(1-\delta^{2}\right) \gamma}{8 \Omega^{\prime} \delta} \sin 2 \Omega^{\prime} t\right] e^{-\gamma t} \\
& +\hbar^{2}\left[\frac{1-\delta^{2}}{4 \delta} \sin 2 \Omega^{\prime} t+\frac{\gamma}{2 \Omega^{\prime}}\left(\operatorname{coth} \frac{\hbar \Omega^{\prime}}{2 k_{B} T}-\frac{1+\delta^{2}}{2 \delta}\right) \sin ^{2} \Omega^{\prime} t\right]^{2} e^{-2 \gamma t}, \\
f_{\text {rel }}(t) & =\frac{\hbar^{2}}{4}\left[1+\frac{1}{4 \delta^{2}}\left(1-\delta^{2}\right)^{2} \sin ^{2} 2 \Omega t\right] .
\end{aligned}
$$

where $\Omega^{\prime}=\sqrt{\Omega^{2}-\gamma^{2} / 4}$ and $\delta=2 \Omega \sigma^{2} / \hbar$. At short times $(t<<1 / \gamma, 1 / \Omega)$,

$$
\begin{aligned}
f_{\mathrm{cm}}(t) & =\frac{\hbar^{2}}{4}\left[1+2\left(\delta \operatorname{coth} \frac{\hbar \Omega^{\prime}}{2 k_{B} T}-1\right) \gamma t\right] \\
f_{\text {rel }}(t) & =\frac{\hbar^{2}}{4} .
\end{aligned}
$$

In this short time span, the time-dependent quantum dispersion of the wave packet constructed in the relative coordinate may be ignored. It is interesting to compare the uncertainty relation (62) with that between the $x_{1}, x_{2}$ and $p_{1}, p_{2}$, denoted by $U_{\mathrm{x}_{\mathrm{i}} \mathrm{p}_{\mathrm{i}}}$ :

$$
U_{\mathrm{x}_{\mathrm{i}} \mathrm{p}_{\mathrm{i}}}=\left(\Delta x_{1}\right)^{2}\left(\Delta p_{1}\right)^{2}\left(\Delta x_{2}\right)^{2}\left(\Delta p_{2}\right)^{2} \geq \frac{1}{8} U(t) .
$$

As will be shown in the next subsection, the variance of the operators $x$ and $X$ etc can indeed provide some useful information about the evolution of quantum entanglement of the Gaussian states.

\section{Dynamics of Entanglement: An Example}

As shown in Subsection IVA, the decoherent effects of a thermal heat bath is captured by the influential functional appearing in (52). An environment that destroys quantum coherence can also disentangle two quantum Brownian particles. The dynamics of decoherence and entanglement of two harmonic oscillators interacting with a common environment is useful for understanding some basic issues in macroscopic quantum phenomena. We will present a more detailed study of this issue in a later paper. Here we show a simple example which has analytic solutions. Take as initial state the Wigner function:

$$
W\left(x_{1}, x_{2}, P_{1}, P_{2}\right)=W_{\mathrm{cm}}(X, P) W_{\text {rel }}(x, p)=e^{-\frac{X^{2}}{2 a^{2}}-\frac{P^{2}}{2 b^{2}}} e^{-\frac{x^{2}}{2 c^{2}}-\frac{p^{2}}{2 d^{2}}} .
$$

where $P, X, x$ and $p$ are canonical variables defined in (5) and (6). We have omitted an irrelevant normalization factor. Note that the widths $a^{2}, b^{2}, c^{2}$ and $d^{2}$ cannot be chosen arbitrarily since they 
have to satisfy the uncertainty relations:

$$
a^{2} b^{2} \geq \frac{\hbar^{2}}{4}, c^{2} d^{2} \geq \frac{\hbar^{2}}{4}
$$

For a wide range of parameters $a, b, c$ and $d$, the Wigner function $W(X, P, x, p)$ is entangled, since generally it cannot be written as a product of $W_{1}\left(x_{1}, P_{1}\right)$ and $W_{2}\left(x_{2}, P_{2}\right)$. At any time $t$, it is known that the separability of the state (68) can be easily detected [36, 37].

Now we consider the dynamics of this state under the influence of a common environment. For greatest simplicity, we assume two free particles coupled to a Markovian thermal bath (Setting $\Omega=0$ and $\kappa=0$ ) and assume the dissipation in $\mathrm{cm}$ coordinates is negligible. Under these conditions, the Wigner equation $W_{\mathrm{cm}}(X, P)$ for cm coordinates (33) takes on a simple form:

$$
\frac{\partial W_{\mathrm{cm}}}{\partial t}=-\frac{P}{M_{1}} \frac{\partial W_{\mathrm{cm}}}{\partial X}+D \frac{\partial^{2} W_{\mathrm{cm}}}{\partial P^{2}}
$$

where $D=2 M_{1} \gamma k_{B} T$. The solution for the dissipative evolution of the center of mass can be easily obtained, and from it, we can compute the variances of $X$ and $P$ at time $t$ to be:

$$
\begin{aligned}
& \left(\Delta X^{2}\right)(t)=\frac{2 D t^{3}}{3 M^{2}}+\frac{b^{2} t^{2}}{4 M^{2}}+a^{2}, \\
& \left(\Delta P^{2}\right)(t)=2 D t+b^{2} .
\end{aligned}
$$

Since the evolution of the Wigner function $W_{\text {rel }}(x, p)$ for the relative coordinates $x, p$ is unitary,

$$
\frac{\partial W_{\mathrm{rel}}}{\partial t}=-\frac{p}{M_{2}} \frac{\partial W_{\mathrm{rel}}}{\partial x}
$$

the variances at $t$ are simply given by

$$
\begin{aligned}
& \left(\Delta x^{2}\right)(t)=\frac{4 d^{2}}{M^{2}} t^{2}+c^{2} \\
& \left(\Delta p^{2}\right)(t)=d^{2}
\end{aligned}
$$

According to [36], we may choose the EPR-like operators as :

$$
u=\tilde{x}_{1}-\tilde{x}_{2}, v=\tilde{P}_{1}+\tilde{P}_{2}
$$

where $\tilde{x}_{i}, \tilde{P}_{i}(i=1,2)$ are the dimensionless variables satisfying $\left[\tilde{x}_{i}, \tilde{P}_{j}\right]=i \delta_{i j}$,

$$
\tilde{x}_{i}=\left(\frac{M D}{\hbar^{3}}\right)^{\frac{1}{4}} x_{i}, \quad \tilde{P}_{i}=\left(\frac{1}{\hbar M D}\right)^{\frac{1}{4}} P_{i}, \quad(i=1,2) .
$$

Then the Gaussian state (68) at $t$ is disentangled if and only if the following inequality is satisfied

$$
\left(\Delta u^{2}\right)(t)+\left(\Delta v^{2}\right)(t) \geq 2
$$


Inserting (72) and (74) into the above inequality, one gets,

$$
A t^{2}+B t+C \geq 2
$$

where

$$
\begin{aligned}
A & =\frac{4 d^{2}}{M^{2}} \sqrt{\frac{M D}{\hbar^{3}}}, \\
B & =2 \sqrt{\frac{D}{\hbar M}}, \\
C & =\frac{b^{2}}{\sqrt{\hbar M D}}+c^{2} \sqrt{\frac{M D}{\hbar^{3}}} .
\end{aligned}
$$

From (79), the disentanglement time $t_{\text {dent }}$ can be determined to be

$$
t_{\mathrm{dent}}=\frac{-B+\sqrt{B^{2}-4 A C+8 A}}{2 A} .
$$

Thus after $t \geq t_{\text {dent }}$ the state (68) becomes completely separable.

In situations when the $2 \mathrm{HO}$ are coupled or share the same environment, it is expected that for some initial states entanglement will persist longer than the case when there is no direct coupling between the two oscillators and each of them is coupled to a separate environment (See, e.g. [38] for two qubits in a common electromagnetic field). This is what one might anticipate would happen for our model in the more general cases. On the other hand, as shown in this simplified example, finite-time disentanglement may yet occur for some initial states when there is no direct coupling between the two oscillators.

Such finite-time decay behavior has been noted before in several cases where two qubits [33] or two harmonic oscillators [34, 39] are individually coupled to their own heat baths. We show here the onset of the finite-time decay for the case of a common heat bath. However, it should be emphasized again that the finite-time disentanglement process found here depends crucially on the choice of initial states because for some initial states the mutual actions between the two harmonic oscillators may lead to entanglement generation. As shown in the case of two-qubits under phase noises, when the initial states are protected by a decoherence-free subspace quantum entanglement is shown to be robust against the thermal noise [40]. The $2 \mathrm{HO}$ model considered here will exhibit similar features, but further details will go beyond the scope of this paper.

\section{FURTHER APPLICATIONS AND DEVELOPMENTS}

a. Summary In this work we derive an exact master equation for two coupled quantum harmonic oscillators interacting via bilinear coupling with a common environment at arbitrary 
temperature made up of many harmonic oscillators with a general spectral density function. We first show a simple derivation based on the observation that the two harmonic oscillator model can be effectively mapped into that of a single harmonic oscillator in a general environment plus a free harmonic oscillator. Since the exact one harmonic oscillator master equation is available [19] the exact master equation with all its coefficients for this two harmonic oscillator model can be easily deduced from the known results of the single harmonic oscillator case. In the second part we give an influence functional treatment of this model and provide explicit expressions for the evolutionary operator of the reduced density matrix which are useful for the study of decoherence and disentanglement issues. We show three applications of this master equation: on the decoherence and disentanglement of two harmonic oscillators due to their interaction with a common environment and a derivation of the uncertainty principle at finite temperature for a composite object, modeled by two interacting harmonic oscillators. For the example of entanglement dynamics under Markovian approximation we find finite-time disentanglement taking place for a Gaussian state.

b. Decoherence and Disentanglement We mention some further developments and applications where our analysis of the $2 \mathrm{HO}$ QBM model can be usefully extended to or compared with. First, for the study of decoherence and disentanglement between two observers, a direct comparison can be carried out with some recent findings in [43] where the model of two harmonic oscillators in relativistic motion (one could be in uniform acceleration) in a common field in Minkowsky or a black hole spacetime. In the latter situation it is of interest to see how entanglement and teleportation will be affected by its unusual causal properties. The case of two oscillators in inertial motion in ordinary Minkowsky spacetime would correspond to our problem here after invoking Lorentz invariance. Second, pursuant to our analysis of the uncertainty principle for composite objects, the substance of our calculations there could be applied to another interesting physical issue pertaining to the Landauer principle [44] and the Clausius inequality. Landauer principle which rests at the foundation of the thermodynamics of information processing, states that (paraphrased in the words of Bennett [45]) "any logically irreversible manipulation of information, such as the erasure of a bit or the merging of two computation paths, must be accompanied by a corresponding entropy increase in non-information bearing degrees of freedom of the information processing apparatus or its environment. Conversely, it is generally accepted that any logically reversible transformation of information can in principle be accomplished by an appropriate physical mechanism operating in a thermodynamically reversible fashion”. (See also [46, 47, 48], the last contains a proposal for a generalized Landauer's principle.) It is well known that the root of this relation is the second law of thermodynamics, but how to measure a logical operation in physical terms or to associate a 
logical state or its transformation with an energy cost and an entropy increase is a new challenge.

c. Quantum Information and Thermodynamics There are many angles to see how Landauer's bound in quantum information theory is related to Clausius' inequality in classical thermodynamics. One such approach is by way of quantum open systems which can treat the dynamics of the system and its quantum information content in fully nonequilibrium settings. This is the conceptual framework and technical systematics we have adopted. Here, dissipation and decoherence in the system and disentanglement between the system and its environment may be followed closely by the evolution of the reduced density matrix (RDM), and the entropy change of the system in the thermodynamic limit may be calculated, with little difficulty. In this vein, using the quantum Brownian model (QBM) of the Caldeira-Leggett (CL) type Hoerhammer and Buettner [50] investigated the influence of entanglement on the relation between the statistical entropy of an open quantum system and the heat exchanged with a low temperature environment. (See also [49]). Their two Brownian oscillator model is of particular relevance to our work here. Compared to the case of a single Brownian particle, two coupled harmonic oscillators can account for how the internal degrees of freedom of the system would affect the heat and entropy changes. Because they adopted the CL treatment their results are subcases of ours here (in the same way that the CL treatment [17] of QBM is related to the HPZ treatment [19], viz, the latter preserves the positive definiteness of RDM in its entire evolution and the HPZ master equation extends the range of validity to non-Markovian regimes.) The CL results are valid only for ohmic baths at high temperatures pertaining to the Markovian regime. For low temperatures and nonOhmic baths pertaining to the nonMarkovian regimes the HPZ treatment is expected to yield more accurate results. Thus using the master equation presented here for the $2 \mathrm{HO}$ QBM model following HPZ treatment and the analytical solutions found recently [51] for various parameter ranges one could obtain an improved Landauer bound for quantum information processing in the nonMarkovian regimes. On the other side of the balance, the Clausius inequality, operative only in the thermodynamic limit, would be too coarse a measure for the energy cost and entropy change of quantum information processing anyway. With the master equations derived here there is much room for tightening the Landauer bound.

d. Qubit-Oscillator Entanglement As subcases of the present model one can investigate the interaction between a two-level system with a harmonic oscillator in a general environment which is of general interest for quantum computer design purposes. One could apply a level reduction scheme such as that used in [41] to one of the two harmonic oscillators, turning the 2HO-bath model into an effective 1HO-spin-boson model where the bimodal oscillator mocks up a qubit. 
The simpler case without an environment would correspond to a two level atom in a multi-mode cavity, such as studied in [42]. Doing a level reduction scheme for both oscillators and viewing the harmonic oscillator bath as a field would reduce our $2 \mathrm{HO}$ QBM model to that of two qubits interacting either directly or indirectly through a common field. An example of the latter situation is studied in [38]. One can use the exact master equations here under appropriate simplifications to describe the nonMarkovian dynamics of such systems.

e. Quantum Superposition of Two Mirrors As mentioned in the Introduction, a new category of problems which has received much attention lately is represented by the quantum superposition of two mirrors [8]. The two mirrors can be modeled by two quantum harmonic oscillators, but in most models for proposed experimental designs, the mirrors are coupled by radiation pressure. This class of models with photon number - mirror displacement (Nx) type of coupling used for mirror-photon entanglement [52], entanglement cooling of a mirror [53] and entanglement of test masses and standard quantum limit [54] is very different from the class with bilinear coupling in QBM studies (Beware of inconsistencies in the usual master equations for this problem, see [55]). On the surface the convenience of the $2 \mathrm{HO}$ model which possesses many useful solutions would not be readily available, but a recent observation by Galley could provide a bridge to these two common classes of models and unleash the resources gathered from the $2 \mathrm{HO}$ QBM problem for the solution of this type of quantum optics problems. (See [56].).

f. Macroscopic Quantum Phenomena Finally, a whole range of issues in macroscopic quantum phenomena can be addressed with the master equation (or the associated Langevin or FokkerPlanck equations) derived here. In particular, decoherence and disentanglement in $2 \mathrm{HO}$ system under more general conditions and $N$-harmonic oscillators systems [23] are currently under study. It can also be applied to the analysis of quantum decoherence, entanglement, fluctuations, dissipation and teleportation of electro- opto-mechanical systems and superposition of moving mirrors due to quantum and radiative effects.

\section{Acknowledgements}

C.-H. C. would like to thank Dr. Kazutomu Shiokawa for discussions on using effective spinboson model in the treatment of quantum entanglement. T. Y. would like to thank Prof. J. H. Eberly for many useful conversations and acknowledges support from ARO Grant W911NF-05-10543 to the University of Rochester. BLH is partially supported by the NSF (PHY-0426696) under the ITR program and by NSA-LPS to the University of Maryland. Part of this work was done 
while we enjoyed the hospitality of the Institute of Physics of the Academia Sinica, Taipei, the National Center for Theoretical Sciences and the Center for Quantum Information Sciences at the National Cheng Kung University, Tainan, Taiwan.

\section{APPENDIX A: DERIVATION OF EXACT MASTER EQUATION FROM PATH INTEGRAL}

Deriving the master equation from the path integral is lengthy, but one of the advantages of this derivation is that the explicit form of the propagator can be used to find an explicit solution of the equation in many interesting cases. We will mainly follow the steps in [19] and outline the key steps in deriving the master equation from the path integral method.

From (35), it is easy to see that, to get the master equation, one first needs to calculate $J_{r}(t+d t, 0)-J_{r}(t, 0)$. The complete derivation can be decomposed into the following four steps.

\section{Step one}

Our first task is to take the functional representation of $J_{r}(t+d t, 0)$ and divide each of the path integrals into two parts. We introduce four intermediate points $x_{1 m}, x_{2 m}, y_{1 m}, y_{2 m}$ at time $t$ and integrate over them, thus symbolically, we write

$$
\int_{0 ; x_{10}}^{t+d t ; x_{1 f}} \mathcal{D} x_{1} \int_{-\infty}^{\infty} d x_{1 m} \int_{0 ; x_{10}}^{t ; x_{1 m}} \mathcal{D} \bar{x}_{1} \int_{t ; x_{1 m}}^{t+d t ; x_{1 f}} \mathcal{D} \tilde{x}_{1} .
$$

There are three similar expressions for the sum over $x_{2}, y_{1}, y_{2}$ histories.

The original histories $x_{1}(\tau)$ are functions defined on $(0, t+d t)$ time interval with $x_{1}(0)=$ $x_{10}, x_{1}(t+d t)=x_{1 f}$. The new set of histories $\bar{x}_{1}(\tau), \tilde{x}_{1}(\tau)$ are functions defined on $(0, t),(t, t+d t)$ intervals with $\bar{x}_{1}(0)=x_{10}, \bar{x}_{1}(t)=x_{1 m}, \tilde{x}_{1}(t)=x_{1 m}, \tilde{x}_{1}(t+d t)=x_{1 f}$.

So we can write

$$
\begin{aligned}
A\left[x_{1}, x_{2}, y_{1}, y_{2}\right] & =S_{S}\left[x_{1}, x_{2}\right]-S_{S}\left[y_{1}, y_{2}\right]+\delta A\left[x_{1}, x_{2}, y_{1}, y_{2}\right] \\
& =A\left[\bar{x}_{1}, \bar{x}_{2}, \bar{y}_{1}, \bar{y}_{2}\right]+A\left[\tilde{x}_{1}, \tilde{x}_{2}, \tilde{y}_{1}, \tilde{y}_{2}\right]+A_{i}\left[\bar{x}_{1}, \bar{x}_{2}, \bar{y}_{1}, \bar{y}_{2}, \tilde{x}_{1}, \tilde{x}_{2}, \tilde{y}_{1}, \tilde{y}_{2}\right],
\end{aligned}
$$

where $A_{i}$ term mixes the $\tilde{x}$ histories with $\bar{x}$ ones. The appearance of the $A_{i}$ term is due to the non-locality of the influence functional. 


\section{Step two}

Next, we will use straight line histories approximation of $\left(\tilde{x}_{1}, \tilde{x}_{2}, \tilde{y}_{1}, \tilde{y}_{2}\right)$. First, note that

$$
\tilde{x}_{1}(s)=x_{1 m}+\left(x_{1 f}-x_{1 m}\right) \frac{s-t}{d t} \equiv x_{1 m}+\beta_{1 x} \frac{s-t}{d t},
$$

and similarly,

$$
\begin{aligned}
& \tilde{x}_{2}(s)=x_{2 m}+\left(x_{2 f}-x_{2 m}\right) \frac{s-t}{d t} \equiv x_{2 m}+\beta_{2 x} \frac{s-t}{d t}, \\
& \tilde{y}_{1}(s)=y_{1 m}+\beta_{1 y} \frac{s-t}{d t}, \quad \tilde{y}_{2}(s)=y_{2 m}+\beta_{2 y} \frac{s-t}{d t} .
\end{aligned}
$$

To compute the time derivative of $J_{r}$, take the limit $d t \rightarrow 0$. Thus we can write

$$
\begin{aligned}
& \left.\prod_{k=1}^{2} \int_{0 ; x_{k 0}}^{t+d t ; x_{k f}} \mathcal{D} x_{k} \int_{0 ; y_{k 0}}^{t+d t ; y_{k f}} \mathcal{D} y_{k} \exp \left(\frac{i}{\hbar} A_{[} x_{1}, x_{2}, y_{1}, y_{2}\right]\right) \\
= & N(t) \prod_{k=1}^{2} \int_{-\infty}^{\infty} d x_{k m} d y_{k m} \exp \left(\frac{i}{\hbar} A\left[\tilde{x}_{1}, \tilde{x}_{2}, \tilde{y}_{1}, \tilde{y}_{2}\right]\right) \\
\times & \prod_{k=1}^{2} \int_{0 ; x_{k 0}}^{t ; x_{k m}} \mathcal{D} \bar{x}_{k} \int_{0 ; y_{k 0}}^{t ; y_{k m}} \mathcal{D} \bar{y}_{k} \exp \left(\frac{i}{\hbar} A\left[\bar{x}_{1}, \bar{x}_{2}, \bar{y}_{1}, \bar{y}_{2}\right]\right) \exp \left(\frac{i}{\hbar} A_{i}\left[\bar{x}_{1}, \bar{x}_{2}, \bar{y}_{1}, \bar{y}_{2}, \tilde{x}_{1}, \tilde{x}_{2}, \tilde{y}_{1}, \tilde{y}_{2}\right]\right)(.
\end{aligned}
$$

Expanding $A$ in $d t$ and keeping the contributions of the first order terms, we get,

$$
A\left[\tilde{x}_{1}, \tilde{x}_{2}, \tilde{y}_{1}, \tilde{y}_{2}\right] \approx \frac{m}{2 d t}\left(\beta_{1 x}^{2}+\beta_{2 x}^{2}-\beta_{1 y}^{2}-\beta_{2 y}^{2}\right)-\frac{1}{2} m \Omega^{2} d t\left(x_{1 f}^{2}+x_{2 f}^{2}-y_{1 f}^{2}-y_{1 f}^{2}\right)+\cdots,
$$

and

$$
\begin{aligned}
A_{i}\left[\bar{x}_{1}, \bar{x}_{2}, \bar{y}_{1}, \bar{y}_{2}, \tilde{x}_{1}, \tilde{x}_{2}, \tilde{y}_{1}, \tilde{y}_{2}\right] \approx & -d t \int_{0}^{t} d s J_{\vec{\Sigma}}(s)\left(\bar{\Sigma}_{1}(s)+\bar{\Sigma}_{2}(s)\right) \\
& +i d t \int_{0}^{t} d s J_{\vec{\Delta}}(s)\left(\bar{\Delta}_{1}(s)+\bar{\Delta}_{2}(s)\right),
\end{aligned}
$$

where

$$
\begin{aligned}
J_{\Sigma_{1}}+J_{\Sigma_{2}} & \equiv J_{\vec{\Sigma}}(s) \frac{2}{d t} \int_{t}^{t+d t} d s^{\prime}\left(\tilde{\Delta}_{1}\left(s^{\prime}\right)+\tilde{\Delta}_{2}\left(s^{\prime}\right)\right) \eta\left(s^{\prime}-s\right) \\
& \approx 2\left(x_{1 f}-y_{1 f}+x_{2 f}-y_{2 f}\right) \eta(t-s)+\cdots
\end{aligned}
$$

and

$$
\begin{aligned}
J_{\Delta_{1}}+J_{\Delta_{2}} & \equiv J_{\vec{\Delta}}(s) \frac{1}{d t} \int_{t}^{t+d t} d s^{\prime}\left(\tilde{\Delta}_{1}\left(s^{\prime}\right)+\tilde{\Delta}_{2}\left(s^{\prime}\right)\right) \nu\left(s^{\prime}-s\right) \\
& \approx\left(x_{1 f}-y_{1 f}+x_{2 f}-y_{2 f}\right) \nu(t-s)+\cdots
\end{aligned}
$$

Here we can keep only terms up to the first order in $\beta_{i}^{2}$. 
In summary, the propagator $J_{r}$ can be formally written as

$$
\begin{aligned}
& J_{r}\left(x_{1 f}, x_{2 f}, y_{1 f}, y_{2 f}, t+d t \mid x_{10}, x_{20}, y_{10}, y_{20}, 0\right) \\
\approx & N(t) \int_{-\infty}^{\infty} d \beta_{1 x} \int_{-\infty}^{\infty} d \beta_{2 x} \int_{-\infty}^{\infty} d \beta_{1 y} \int_{-\infty}^{\infty} d \beta_{2 y} \exp \left(\frac{i m}{2 \hbar d t}\left(\beta_{1 x}^{2}+\beta_{2 x}^{2}-\beta_{1 y}^{2}-\beta_{2 y}^{2}\right)\right) \\
\times & \left\{1-\frac{i}{\hbar} d t\left[V\left(x_{1 f}, x_{2 f}\right)-V\left(y_{1 f}, y_{2 f}\right)\right]\right\} \\
& \times \tilde{J}_{r}\left(x_{1 m}, x_{2 m}, y_{1 m}, y_{2 m}, t+d t \mid x_{10}, x_{20}, y_{10}, y_{20}, 0 ;[\vec{b}]\right),
\end{aligned}
$$

where

$$
\begin{aligned}
& \tilde{J}_{r}\left(x_{1 m}, x_{2 m}, y_{1 m}, y_{2 m}, t+d t \mid x_{10}, x_{20}, y_{10}, y_{20}, 0 ;[\vec{b}]\right)= \\
& \int_{0 ; x_{10}}^{t ; x_{1 m}} \mathcal{D} \bar{x}_{1} \int_{0 ; x_{20}}^{t ; x_{2 m}} \mathcal{D} \bar{x}_{2} \int_{0 ; y_{10}}^{t ; y_{1 m}} \mathcal{D} \bar{y}_{1} \int_{0 ; y_{20}}^{t ; y_{2 m}} \mathcal{D} \bar{y}_{2} \exp \left(\frac{i}{\hbar} A\left[\bar{x}_{1}, \bar{x}_{2}, \bar{y}_{1}, \bar{y}_{2}\right]\right) \\
& \exp \left[\frac{i}{\hbar}\left(-d t \int_{0}^{t} d s J_{\vec{\Sigma}}(s)\left(\bar{\Sigma}_{1}(s)+\bar{\Sigma}_{2}(s)\right)+i d t \int_{0}^{t} d s J_{\vec{\Delta}}(s)\left(\bar{\Delta}_{1}(s)+\bar{\Delta}_{2}(s)\right)\right)\right]
\end{aligned}
$$

and

$$
\vec{b}=\left(\begin{array}{c}
J_{\vec{\Sigma}} \\
J_{\vec{\Delta}}
\end{array}\right)
$$

where the sources $\vec{b}$ are functions of the end points. Note that $\tilde{J}_{r}(\vec{b})$ can be interpreted as the evolution operator under the action of two external sources.

\section{Step three}

Computation of the path integral $\tilde{J}_{r}(\vec{b})$ can be done as follows. First, one can show that

$$
\begin{aligned}
& \tilde{J}_{r}\left(x_{1 m}, x_{2 m}, y_{1 m}, y_{2 m}, t \mid x_{10}, x_{20}, y_{10}, y_{20}, 0 ;[\vec{b}]\right)= \\
& J_{r}\left(x_{1 m}, x_{2 m}, y_{1 m}, y_{2 m}, t \mid x_{10}, x_{20}, y_{10}, y_{20}, 0\right) W\left(x_{1 m}, x_{2 m}, y_{1 m}, y_{2 m}, x_{10}, x_{20}, y_{10}, y_{20}, d t\right)
\end{aligned}
$$

(Note that the function $J_{r}$ is the evolution operator without source while the function $W$ is a function of the end points. )

Then we may parametrize the paths, and write

$$
\begin{aligned}
& \Sigma_{1}(s)=\varphi_{1}(s)+\Sigma_{c l, 1}(s), \quad \Sigma_{2}(s)=\varphi_{2}(s)+\Sigma_{c l, 2}(s) \\
& \Delta_{1}(s) \psi_{1}(s)+\Delta_{c l, 1}(s), \quad \Delta_{2}(s)=\psi_{2}(s)+\Delta_{c l, 2}(s)
\end{aligned}
$$

where the "classical paths" $\left(\begin{array}{c}\Sigma \\ \Delta\end{array}\right)_{c l}$ are the solutions to the equation of motion derived from the real part of $A\left[\Sigma_{1}, \Sigma_{2}, \Delta_{1}, \Delta_{2}\right]$. 
After this path reparametrization and making a saddle point approximation, this path integral $\tilde{J}_{r}\left(x_{1 m}, x_{2 m}, y_{1 m}, y_{2 m}, t \mid x_{10}, x_{20}, y_{10}, y_{20}, 0 ;[\vec{b}]\right)$ can be written as

$$
\begin{aligned}
& \tilde{J}_{r}\left(x_{1 m}, x_{2 m}, y_{1 m}, y_{2 m}, t \mid x_{10}, x_{20}, y_{10}, y_{20}, 0 ;[\vec{b}]\right)=\tilde{J}_{r}(0,0,0,0, t \mid 0,0,0,0,0 ;[\vec{b}]) \\
\times & \exp \left(\frac{i}{\hbar} A\left[\Sigma_{c l, 1}, \Sigma_{c l, 2}, \Delta_{c l, 1}, \Delta_{c l, 2}\right]\right) \\
\times & \exp \left[\frac{i}{\hbar}\left(-d t \int_{0}^{t} d s J_{\vec{\Sigma}}(s)\left(\Sigma_{c l, 1}(s)+\Sigma_{c l, 2}(s)\right)+i d t \int_{0}^{t} d s J_{\vec{\Delta}}(s)\left(\Delta_{c l, 1}(s)+\Delta_{c l, 2}(s)\right)\right)\right],
\end{aligned}
$$

where

$$
\begin{aligned}
& \tilde{J}_{r}(0,0,0,0, t \mid 0,0,0,0,0 ;[\vec{b}]) \int_{0 ; \varphi_{1}=0}^{t ; \varphi_{1}=0} \mathcal{D} \varphi_{1} \int_{0 ; \varphi_{2}=0}^{t ; \varphi_{2}=0} \mathcal{D} \varphi_{2} \int_{0 ; \psi_{1}=0}^{t ; \psi_{1}=0} \mathcal{D} \psi_{1} \int_{0 ; \psi_{2}=0}^{t ; \psi_{2}=0} \mathcal{D} \psi_{2} \\
& \exp \left\{i\left[\int_{0}^{t} d s_{1} \int_{0}^{t} \frac{1}{2} \Psi^{T}\left(s_{1}\right) \hat{O}\left(s_{1}, s_{2}\right) \Psi\left(s_{2}\right)+\int_{0}^{t} d s \Psi^{T}(s) \cdot \vec{B}(s)\right]\right\} .
\end{aligned}
$$

Note that

$$
\Psi=\left(\begin{array}{c}
\Psi_{1} \\
\Psi_{2}
\end{array}\right)=\left(\begin{array}{l}
\varphi_{1} \\
\psi_{1} \\
\varphi_{2} \\
\psi_{2}
\end{array}\right)
$$

and

$$
\vec{B}=\left(\begin{array}{c}
-d t J_{\vec{\Sigma}} \\
i d t J_{\vec{\Delta}}+i \tilde{J}_{\vec{\Delta}} \\
-d t J_{\vec{\Sigma}} \\
i d t J_{\vec{\Delta}}+i \tilde{J}_{\vec{\Delta}}
\end{array}\right)
$$

where $\tilde{J}_{\vec{\Delta}}$ is a new source which appears because the nonlocality of the influence functional. It couples the classical paths to the $\Psi$ paths.

$$
\tilde{J}_{\vec{\Delta}}(s)=\int_{0}^{t} d s^{\prime}\left[\Delta_{c l, 1}\left(s^{\prime}\right)+\Delta_{c l, 2}\left(s^{\prime}\right)\right] \nu\left(s-s^{\prime}\right) .
$$

The matrix operator $\hat{O}\left(s_{1}, s_{2}\right)$ is defined as follows:

$$
\begin{gathered}
O_{11}\left(s_{1}, s_{2}\right)=O_{33}\left(s_{1}, s_{2}\right)=O_{13}\left(s_{1}, s_{2}\right) O_{31}\left(s_{1}, s_{2}\right)=0, \\
O_{22}\left(s_{1}, s_{2}\right)=O_{44}\left(s_{1}, s_{2}\right)=O_{24}\left(s_{1}, s_{2}\right) O_{42}\left(s_{1}, s_{2}\right)=2 i \nu\left(s_{1}-s_{2}\right), \\
O_{14}\left(s_{1}, s_{2}\right)=O_{32}\left(s_{1}, s_{2}\right)=2 \theta\left(s_{2}-s_{1}\right) \eta\left(s_{1}-s_{2}\right),
\end{gathered}
$$




$$
\begin{gathered}
O_{41}\left(s_{1}, s_{2}\right)=O_{23}\left(s_{1}, s_{2}\right)=2 \theta\left(s_{1}-s_{2}\right) \eta\left(s_{1}-s_{2}\right), \\
O_{12}\left(s_{1}, s_{2}\right)=O_{34}\left(s_{1}, s_{2}\right)=\left(\frac{d^{2}}{d s_{1}^{2}}+\Omega^{2}\right) \delta\left(s_{1}-s_{2}\right)+2 \theta\left(s_{2}-s_{1}\right) \eta\left(s_{1}-s_{2}\right), \\
O_{21}\left(s_{1}, s_{2}\right)=O_{43}\left(s_{1}, s_{2}\right)=\left(\frac{d^{2}}{d s_{1}^{2}}+\Omega^{2}\right) \delta\left(s_{1}-s_{2}\right)+2 \theta\left(s_{1}-s_{2}\right) \eta\left(s_{1}-s_{2}\right) .
\end{gathered}
$$

The Gaussian path integral can be computed in terms of the inverse of the operator $\hat{O}$, which is given by $\hat{G} \equiv \hat{O}^{-1}$. Hence to first order in $d t$, we have

$$
\begin{aligned}
& \tilde{J}_{r}(0,0,0,0, t \mid 0,0,0,0,0 ;[\vec{b}]) \int_{0 ; \varphi_{1}=0}^{t ; \varphi_{1}=0} \mathcal{D} \varphi_{1} \int_{0 ; \varphi_{2}=0}^{t ; \varphi_{2}=0} \mathcal{D} \varphi_{2} \int_{0 ; \psi_{1}=0}^{t ; \psi_{1}=0} \mathcal{D} \psi_{1} \int_{0 ; \psi_{2}=0}^{t ; \psi_{2}=0} \mathcal{D} \psi_{2} \\
& \exp \left\{i\left[\int_{0}^{t} d s_{1} \int_{0}^{t} \frac{1}{2} \Psi^{T}\left(s_{1}\right) \hat{O}\left(s_{1}, s_{2}\right) \Psi\left(s_{2}\right)+\int_{0}^{t} d s \Psi^{T}(s) \cdot \vec{B}(s)\right]\right\} \\
& =\int \mathcal{D} \varphi_{1} \int \mathcal{D} \varphi_{2} \int \mathcal{D} \psi_{1} \int \mathcal{D} \psi_{2} \exp \left\{i\left[\frac{1}{2}\left(\Psi^{T}+\vec{B}^{T} \cdot \hat{O}^{-1}\right) \hat{O}\left(\Psi+\hat{O}^{-1} \cdot \vec{B}\right)-\frac{1}{2} \vec{B}^{T} \hat{O}^{-1} \vec{B}\right]\right\} \\
= & Z_{0}(t) \exp \left\{-\frac{i}{2} \vec{B}^{T} \hat{O}^{-1} \vec{B}\right\} \\
\approx & Z_{0}(t)\left(1-\frac{i}{2} \vec{B}^{T} \hat{O}^{-1} \vec{B}\right) \\
\approx & Z_{0}(t)\left(1-d t \int_{0}^{t} d s_{1} \int_{0}^{t} d s_{2} J_{\vec{\Sigma}}\left(s_{1}\right)\left[G_{12}\left(s_{1}, s_{2}\right)+G_{14}\left(s_{1}, s_{2}\right)+G_{21}\left(s_{2}, s_{1}\right)+G_{41}\left(s_{1}, s_{2}\right)\right] \tilde{J}_{\vec{\Delta}}\left(s_{2}\right)\right) .
\end{aligned}
$$

Note that the Green's function $\left(G_{12}+G_{32}\right) \equiv \tilde{G}_{12}\left(s_{1}, s_{2}\right)$ satisfies the following equation

$$
\frac{d^{2}}{d s_{1}^{2}} \tilde{G}_{12}\left(s_{1}, s_{2}\right)+\Omega^{2} \tilde{G}_{12}\left(s_{1}, s_{2}\right)+4 \int_{0}^{s_{1}} d \tau \eta\left(s_{1}-\tau\right) \tilde{G}_{12}\left(s_{1}, \tau\right)=\delta\left(s_{1}-s_{2}\right)
$$

with boundary conditions $\tilde{G}_{12}\left(0, s_{2}\right)=\tilde{G}_{12}\left(s_{1}, t\right)=0$. The equation for $\left(G_{21}+G_{23}\right) \equiv \tilde{G}_{21}\left(s_{1}, s_{2}\right)$ are analogous.

Now we can show that

$$
\begin{aligned}
& \tilde{J}_{r}\left(x_{1 m}, x_{2 m}, y_{1 m}, y_{2 m}, t \mid x_{10}, x_{20}, y_{10}, y_{20}, 0 ;[\vec{b}]\right) \\
& =\tilde{J}_{r}(0,0,0,0, t \mid 0,0,0,0,0 ;[\vec{b}]) \exp \left\{i\left(A\left[\Sigma_{c l, 1}, \Sigma_{c l, 2}, \Delta_{c l, 1}, \Delta_{c l, 2}\right]\right)\right\} \\
& \times \exp \left\{i\left(-d t \int_{0}^{t} d s J_{\vec{\Sigma}}(s)\left(\Sigma_{c l, 1}(s)+\Sigma_{c l, 2}(s)\right)+i d t \int_{0}^{t} d s J_{\vec{\Delta}}(s)\left(\Delta_{c l, 1}(s)+\Delta_{c l, 2}(s)\right)\right)\right\} \\
& \approx Z_{0}(t) \exp \left\{i A\left[\Sigma_{c l, 1}, \Sigma_{c l, 2}, \Delta_{c l, 1}, \Delta_{c l, 2}\right]\right\} \\
& \times\left\{1-d t \int_{0}^{t} d s_{1} \int_{0}^{t} d s_{2} J_{\vec{\Sigma}}\left(s_{1}\right)\left[\tilde{G}_{12}\left(s_{1}, s_{2}\right)+\tilde{G}_{21}\left(s_{1}, s_{2}\right)\right] \tilde{J}_{\vec{\Delta}}\left(s_{2}\right)\right. \\
& \left.-i d t \int_{0}^{t} d s J_{\vec{\Sigma}}(s)\left(\Sigma_{c l, 1}(s)+\Sigma_{c l, 2}(s)\right)+(i)^{2} d t \int_{0}^{t} d s J_{\vec{\Delta}}(s)\left(\Delta_{c l, 1}(s)+\Delta_{c l, 2}(s)\right)\right\} \\
& =J_{r}\left(x_{1 m}, x_{2 m}, y_{1 m}, y_{2 m}, t \mid x_{10}, x_{20}, y_{10}, y_{20}, 0\right) \\
& \times W\left(x_{1 m}, x_{2 m}, y_{1 m}, y_{2 m}, x_{10}, x_{20}, y_{10}, y_{20}, d t\right)
\end{aligned}
$$


where $W$ is given by,

$$
\begin{aligned}
& W\left(x_{1 m}, x_{2 m}, y_{1 m}, y_{2 m}, x_{10}, x_{20}, y_{10}, y_{20}, d t\right) \\
= & 1-i d t\left[\int_{0}^{t} d s 2\left(\Delta_{1 f}+\Delta_{2 f}\right) \eta(t-s) u_{1}(s) \tilde{\Sigma}_{c l}(0)\right. \\
& \left.+\int_{0}^{t} d s 2\left(\Delta_{1 f}+\Delta_{2 f}\right) \eta(t-s) u_{2}(s) \tilde{\Sigma}_{c l}(t)\right] \\
- & d t\left[\int_{0}^{t} d s\left(\Delta_{1 f}+\Delta_{2 f}\right) \nu(t-s) v_{1}(s) \tilde{\Delta}_{c l}(0)+\int_{0}^{t} d s\left(\Delta_{1 f}+\Delta_{2 f}\right) \nu(t-s) v_{2}(s) \tilde{\Delta}_{c l}(t)\right] \\
- & d t\left[\int_{0}^{t} d s_{1} \int_{0}^{t} d s_{2} \int_{0}^{t} d s_{3} 2\left(\Delta_{1 f}+\Delta_{2 f}\right) \eta\left(t-s_{1}\right)\left[\tilde{G}_{12}\left(s_{1}, s_{2}\right)+\tilde{G}_{21}\left(s_{2}, s_{1}\right)\right]\right. \\
& \times \nu\left(s_{2}-s_{3}\right) v_{1}\left(s_{3}\right) \tilde{\Delta}_{c l}(0) \\
+ & \int_{0}^{t} d s_{1} \int_{0}^{t} d s_{2} \int_{0}^{t} d s_{3} 2\left(\Delta_{1 f}+\Delta_{2 f}\right) \eta\left(t-s_{1}\right)\left[\tilde{G}_{12}\left(s_{1}, s_{2}\right)\right. \\
& \left.\left.+\tilde{G}_{21}\left(s_{2}, s_{1}\right)\right] \nu\left(s_{2}-s_{3}\right) v_{2}\left(s_{3}\right) \tilde{\Delta}_{c l}(t)\right] .
\end{aligned}
$$

To simplify the expressions, let us define

$$
\begin{aligned}
& d_{1}(t)=2 \int_{0}^{t} d s \eta(t-s) u_{1}(s), \quad d_{2}(t)=2 \int_{0}^{t} d s \eta(t-s) u_{2}(s) \\
& c_{1}(t)=\int_{0}^{t} d s_{1} \int_{0}^{t} d s_{2} \int_{0}^{t} d s_{3} \eta\left(t-s_{1}\right)\left[\tilde{G}_{12}\left(s_{1}, s_{2}\right)+\tilde{G}_{21}\left(s_{2}, s_{1}\right)\right] \nu\left(s_{2}-s_{3}\right) v_{1}\left(s_{3}\right), \\
& c_{2}(t)=\int_{0}^{t} d s_{1} \int_{0}^{t} d s_{2} \int_{0}^{t} d s_{3} \eta\left(t-s_{1}\right)\left[\tilde{G}_{12}\left(s_{1}, s_{2}\right)+\tilde{G}_{21}\left(s_{2}, s_{1}\right)\right] \nu\left(s_{2}-s_{3}\right) v_{2}\left(s_{3}\right), \\
& e_{1}(t)=\int_{0}^{t} d s \nu(t-s) v_{2}(s)=\int_{0}^{t} d s \nu(t-s) u_{1}(t-s)=\int_{0}^{t} d s \nu(s) u_{1}(s) \\
& e_{2}(t)=\int_{0}^{t} d s \nu(t-s) v_{1}(s)=\int_{0}^{t} d s \nu(t-s) u_{2}(t-s)=\int_{0}^{t} d s \nu(s) u_{2}(s) .
\end{aligned}
$$

Finally, we have,

$$
\begin{aligned}
& J_{r}\left(x_{1 f}, x_{2 f}, y_{1 f}, y_{2 f}, t+d t \mid x_{10}, x_{20}, y_{10}, y_{20}, 0\right) \\
= & N(t) \prod_{k=1}^{2} \int_{-\infty}^{\infty} d \beta_{k x} \int_{-\infty}^{\infty} d \beta_{k y} \exp \left(\frac{i}{2 d t}\left(\beta_{1 x}^{2}+\beta_{2 x}^{2}-\beta_{1 y}^{2}-\beta_{2 y}^{2}\right)\right) \\
\times & \left\{1-d t\left[i\left(V\left(x_{1 f}, x_{2 f}\right)-V\left(y_{1 f}, y_{2 f}\right)\right)+i\left(\Delta_{1 f}+\Delta_{2 f}\right)\left(d_{1}(t)\left(\Sigma_{i, 1}+\Sigma_{i, 2}\right)\right.\right.\right. \\
& \left.+d_{2}(t)\left(\Sigma_{1 f}+\Sigma_{2 f}\right)\right)+\left(\Delta_{1 f}+\Delta_{2 f}\right)\left(\Delta_{i, 1}+\Delta_{i, 2}\right)\left(e_{2}(t)+2 c_{1}(t)\right) \\
& \left.\left.+\left(\Delta_{1 f}+\Delta_{2 f}\right)^{2}\left(e_{1}(t)+2 c_{2}(t)\right)\right]\right\} \\
& \times\left\{J_{r}+\frac{1}{2}\left[\frac{\partial^{2} J_{r}}{\partial x_{1 f}^{2}}\left(-\beta_{1 x}\right)^{2}+\frac{\partial^{2} J_{r}}{\partial x_{2 f}^{2}}\left(-\beta_{2 x}\right)^{2}+\frac{\partial^{2} J_{r}}{\partial y_{1 f}^{2}}\left(-\beta_{1 y}\right)^{2}+\frac{\partial^{2} J_{r}}{\partial y_{2 f}^{2}}\left(-\beta_{2 y}\right)^{2}\right]\right\} .
\end{aligned}
$$


Hence

$$
\begin{aligned}
& J_{r}(t+d t)-J_{r}=-d t J_{r}\left[i \frac{1}{2} \Omega^{2}\left(x_{1 f}^{2}+x_{2 f}^{2}-y_{1 f}^{2}-y_{2 f}^{2}\right)+\left(\Delta_{1 f}+\Delta_{2 f}\right) \times\right. \\
& {\left[i\left(d_{1}(t)\left(\Sigma_{i, 1}+\Sigma_{i, 2}\right)+d_{2}(t)\left(\Sigma_{1 f}+\Sigma_{2 f}\right)\right)+\left(\Delta_{i, 1}+\Delta_{i, 2}\right)\left(e_{2}(t)+2 c_{1}(t)\right)\right.} \\
& \left.\left.+\left(\Delta_{1 f}+\Delta_{2 f}\right)\left(e_{1}(t)+2 c_{2}(t)\right)\right]\right] \\
& +\frac{1}{2} \frac{d t}{-i} \frac{\partial^{2} J_{r}}{\partial x_{1 f}^{2}}+\frac{1}{2} \frac{d t}{-i} \frac{\partial^{2} J_{r}}{\partial x_{2 f}^{2}}+\frac{1}{2} \frac{d t}{i} \frac{\partial^{2} J_{r}}{\partial y_{1 f}^{2}}+\frac{1}{2} \frac{d t}{i} \frac{\partial^{2} J_{r}}{\partial y_{2 f}^{2}}
\end{aligned}
$$

We can then get the evolution equation for the propagator $J_{r}$.

$$
\begin{aligned}
& i \frac{\partial}{\partial t} J_{r}\left(x_{1 f}, x_{2 f}, y_{1 f}, y_{2 f}, t \mid x_{10}, x_{20}, y_{10}, y_{20}, 0\right) \\
= & i \frac{\partial}{\partial t}\left[J_{r}\left(x_{1 f}, x_{2 f}, y_{1 f}, y_{2 f}, t+d t \mid x_{10}, x_{20}, y_{10}, y_{20}, 0\right)-J_{r}\left(x_{1 f}, x_{2 f}, y_{1 f}, y_{2 f}, t \mid x_{10}, x_{20}, y_{10}, y_{20}, 0\right)\right] \\
= & \left\{-\frac{1}{2}\left(\frac{\partial^{2}}{\partial x_{1 f}^{2}}+\frac{\partial^{2}}{\partial x_{2 f}^{2}}-\frac{\partial^{2}}{\partial y_{1 f}^{2}}-\frac{\partial^{2}}{\partial y_{2 f}^{2}}\right)+\frac{1}{2} \Omega^{2}\left(x_{1 f}^{2}+x_{2 f}^{2}-y_{1 f}^{2}-y_{2 f}^{2}\right)\right. \\
+ & \left(\Delta_{1 f}+\Delta_{2 f}\right)\left(\left(d_{1}(t)\left(\Sigma_{i, 1}+\Sigma_{i, 2}\right)+d_{2}(t)\left(\Sigma_{1 f}+\Sigma_{2 f}\right)\right)\right) \\
- & i\left(\Delta_{1 f}+\Delta_{2 f}\right)\left(\Delta_{i, 1}+\Delta_{i, 2}\right)\left(e_{2}(t)+2 c_{1}(t)\right) \\
- & \left.i\left(\Delta_{1 f}+\Delta_{2 f}\right)^{2}\left(e_{1}(t)+2 c_{2}(t)\right)\right\} J_{r}\left(x_{1 f}, x_{2 f}, y_{1 f}, y_{2 f}, t \mid x_{10}, x_{20}, y_{10}, y_{20}, 0\right) .
\end{aligned}
$$

\section{Step four}

Now we have the explicit expression for $J_{r}$. But we still need to deal with terms of the form like $\Delta_{1 i} J$. To do so we can differentiate $J$ with respect to $\Sigma_{1 f}$ and get

$$
\begin{aligned}
\partial_{\Sigma_{1 f}} J= & {\left[i b_{1}(t)\left(\Delta_{1 f}+\Delta_{2 f}\right)+i b_{5}(t)\left(\Delta_{1 f}\right.\right.} \\
& \left.\left.-\Delta_{2 f}\right)-i b_{3}(t)\left(\Delta_{1 i}+\Delta_{2 i}\right)-i b_{7}(t)\left(\Delta_{1 i}-\Delta_{2 i}\right)\right] J .
\end{aligned}
$$

Similarly if we want $\Delta_{2 i} J$, we can differentiate $J$ with respect to $\Sigma_{2 f}$ and get

$$
\begin{aligned}
\partial_{\Sigma_{2 f}} J= & {\left[i b_{1}(t)\left(\Delta_{1 f}+\Delta_{2 f}\right)-i b_{5}(t)\left(\Delta_{1 f}\right.\right.} \\
& \left.\left.-\Delta_{2 f}\right)-i b_{3}(t)\left(\Delta_{1 i}+\Delta_{2 i}\right)+i b_{7}(t)\left(\Delta_{1 i}-\Delta_{2 i}\right)\right] J
\end{aligned}
$$

The sum of these two equations gives

$$
\left(\partial_{\Sigma_{1 f}}+\partial_{\Sigma_{2 f}}\right) J=\left[2 i b_{1}(t)\left(\Delta_{1 f}+\Delta_{2 f}\right)-2 i b_{3}(t)\left(\Delta_{1 i}+\Delta_{2 i}\right)\right] J
$$

This can be written as

$$
\left(\Delta_{1 i}+\Delta_{2 i}\right) J=\frac{1}{2 b_{3}(t)}\left[i\left(\partial_{\Sigma_{1 f}}+\partial_{\Sigma_{2 f}}\right)+2 b_{1}(t)\left(\Delta_{1 f}+\Delta_{2 f}\right)\right] J
$$


Similarly, we can differentiate with respect to $\Delta_{1 f}$ (or $\Delta_{2 f}$ ) to get $\Sigma_{1 i} J$ (or $\Sigma_{2 i} J$ ). The sum of these two equations gives

$$
\begin{aligned}
\left(\partial_{\Delta_{1 f}}+\partial_{\Delta_{2 f}}\right) J & =2\left[i b_{2}(t)\left(\Sigma_{1 i}+\Sigma_{2 i}\right)+i b_{1}(t)\left(\Sigma_{1 f}+\Sigma_{2 f}\right)\right. \\
& \left.-a_{12}(t)\left(\Delta_{1 i}+\Delta_{2 i}\right)-2 a_{11}(t)\left(\Delta_{1 f}+\Delta_{2 f}\right)\right] J
\end{aligned}
$$

and

$$
\begin{aligned}
\left(\Sigma_{1 i}+\Sigma_{2 i}\right) J= & \frac{1}{2 b_{2}(t)}\left[-i\left(\partial_{\Delta_{1 f}}+\partial_{\Delta_{2 f}}\right)+\frac{a_{12}(t)}{b_{3}(t)}\left(\partial_{\Sigma_{1 f}}+\partial_{\Sigma_{2 f}}\right)-2 b_{1}(t)\left(\Sigma_{1 f}+\Sigma_{2 f}\right)\right. \\
& \left.-i\left[4 a_{11}(t)+2 \frac{a_{12}(t) b_{1}(t)}{b_{3}(t)}\right]\left(\Delta_{1 f}+\Delta_{2 f}\right)\right] J
\end{aligned}
$$

Substituting in what we already have for $\left(\Sigma_{1 i}+\Sigma_{2 i}\right) J$ and $\left(\Delta_{1 i}+\Delta_{2 i}\right) J$, and multiplying by $\rho_{0}$ and integrating over initial coordinates, we obtain

$$
\begin{aligned}
& \left(\Delta_{1 f}+\Delta_{2 f}\right) d_{1}(t)\left(\Sigma_{1 i}+\Sigma_{2 i}\right) J \\
= & \left(\Delta_{1 f}+\Delta_{2 f}\right) d_{1}(t)\left[\frac{-i}{2 b_{2}(t)}\left(\partial_{\Delta_{1 f}}+\partial_{\Delta_{2 f}}\right)+\frac{a_{12}(t)}{2 b_{2}(t) b_{3}(t)}\left(\partial_{\Sigma_{1 f}}+\partial_{\Sigma_{2 f}}\right)\right. \\
- & \left.\frac{b_{1}(t)}{b_{2}(t)}\left(\Sigma_{1 f}+\Sigma_{2 f}\right)-i\left[\frac{2 a_{11}(t)}{b_{2}(t)}+\frac{a_{12}(t) b_{1}(t)}{b_{2}(t) b_{3}(t)}\right]\left(\Delta_{1 f}+\Delta_{2 f}\right)\right] J,
\end{aligned}
$$

and

$$
\begin{aligned}
& \left(\Delta_{1 f}+\Delta_{2 f}\right)\left(e_{2}(t)+2 c_{1}(t)\right)\left(\Delta_{1 i}+\Delta_{2 i}\right) J \\
= & \left(\Delta_{1 f}+\Delta_{2 f}\right)\left(e_{2}(t)+2 c_{1}(t)\right)\left[\frac{i}{2 b_{3}(t)}\left(\partial_{\Sigma_{1 f}}+\partial_{\Sigma_{2 f}}\right)+\frac{b_{1}(t)}{b_{3}(t)}\left(\Delta_{1 f}+\Delta_{2 f}\right)\right] J
\end{aligned}
$$

Hence we can write the evolution equation for the reduced density matrix as

$$
\begin{aligned}
i \frac{\partial}{\partial t} \rho_{r}= & {\left[-\frac{1}{2}\left(\frac{\partial^{2}}{\partial x_{1}^{2}}+\frac{\partial^{2}}{\partial x_{2}^{2}}-\frac{\partial^{2}}{\partial y_{1}^{2}}-\frac{\partial^{2}}{\partial y_{2}^{2}}\right)+\frac{1}{2} \Omega^{2}\left(x_{1}^{2}+x_{2}^{2}-y_{1}^{2}-y_{2}^{2}\right)\right] \rho_{r} } \\
& +\delta \Omega^{2}(t)\left(\Delta_{1 f}+\Delta_{2 f}\right)\left(\Sigma_{1 f}+\Sigma_{2 f}\right) \rho_{r} \\
& -i A_{1}(t)\left(\Delta_{1 f}+\Delta_{2 f}\right)\left(\partial_{\Delta_{1 f}}+\partial_{\Delta_{2 f}}\right) \rho_{r} \\
& -i A_{2}(t)\left(\Delta_{1 f}+\Delta_{2 f}\right)^{2} \rho_{r} \\
& +A_{3}(t)\left(\Delta_{1 f}+\Delta_{2 f}\right)\left(\partial_{\Sigma_{1 f}}+\partial_{\Sigma_{2 f}}\right) \rho_{r}
\end{aligned}
$$

where

$$
\frac{\partial}{\partial \Sigma}=\frac{\partial}{\partial x}+\frac{\partial}{\partial y} ; \quad \frac{\partial}{\partial \Delta}=\frac{1}{2}\left(\frac{\partial}{\partial x}-\frac{\partial}{\partial y}\right)
$$

and

$$
\delta \Omega^{2}(t) \equiv d_{2}(t)-d_{1}(t) \frac{b_{1}(t)}{b_{2}(t)}, \quad A_{1}(t) \equiv \frac{d_{1}(t)}{2 b_{2}(t)}
$$




$$
\begin{gathered}
A_{2}(t) \equiv d_{1}(t)\left[\frac{2 a_{11}(t)}{b_{2}(t)}+\frac{a_{12}(t) b_{1}(t)}{b_{2}(t) b_{3}(t)}\right]+\left(e_{1}(t)+2 c_{2}(t)\right)+\left(e_{2}(t)+2 c_{1}(t)\right) \frac{b_{1}(t)}{b_{3}(t)} \\
A_{3}(t) \equiv \frac{d_{1}(t) a_{12}(t)}{2 b_{2}(t) b_{3}(t)}+\frac{e_{2}(t)+2 c_{1}(t)}{2 b_{3}(t)} .
\end{gathered}
$$

This immediately leads to the general master equation (26).

\section{Coefficients of the Master Equation}

The determination of the coefficients is reasonably standard, so we only provide the explicit forms of those time-dependent functions that will be used later on. As shown in [19], the functions $\delta \Omega^{2}(t), \Gamma(t), \Delta(t), \Sigma(t)$ can be constructed in terms of the elementary functions $u_{i}(s), i=1,2$, which satisfy the following homogeneous integro-differential equation:

$$
\ddot{f}(s)+\Omega^{2} f(s)+\frac{4}{M} \int_{0}^{s} d \lambda \eta(s-\lambda) f(\lambda)=0
$$

with the boundary conditions:

$$
u_{1}(s=0)=1, u_{1}(s=t)=0
$$

and

$$
u_{2}(s=0)=0, u_{2}(s=t)=1 \text {. }
$$

Here $\eta(t-s)$ is the dissipation kernel given by

$$
\eta(s)=-\int_{0}^{\infty} d \omega I(\omega) \sin (\omega s)
$$

and $I(\omega)$ is the spectral density of the environment. Note that the numerical factor 4 before the integral in this equation is different from that in [19]. This is the main difference due to the presence of two harmonic oscillators. Although the two harmonic oscillators are not coupled directly, they are connected by the common reservoir, hence they affect each other dynamically.

Let $G_{1}(s, \tau)$ be the Green function obeying the following equation:

$$
\frac{d^{2}}{d s^{2}} G_{1}(s, \tau)+\Omega^{2} G_{1}(s, \tau)+\frac{4}{M} \int_{0}^{s} d \tau \eta(s-\tau) G_{1}(s, \tau)=\delta(s-\tau),
$$

with initial conditions:

$$
G_{1}(s=0, \tau)=0,\left.\frac{d}{d s} G_{1}(s, \tau)\right|_{s=0}=0
$$


The Green function $G_{2}(s, \tau)$ is defined analogously. The coefficients can then be written as

$$
\begin{gathered}
\delta \Omega^{2}(t)=\frac{2}{M} \int_{0}^{t} d s \eta(t-s)\left(u_{2}(s)-\frac{u_{1}(s) \dot{u}_{2}(t)}{\dot{u}_{1}(t)}\right), \\
\Gamma(t)=\frac{1}{M} \int_{0}^{t} d s \eta(t-s) \frac{u_{1}(s)}{\dot{u}_{1}(t)} \\
\Delta(t)=\frac{\hbar}{2 M} \int_{0}^{t} d \lambda G_{1}(t, \lambda) \nu(t-\lambda) \\
-\frac{4 \hbar}{M^{2}} \int_{0}^{t} d s \int_{s}^{t} d \tau \int_{0}^{t} d \lambda \eta(t-s) G_{1}(t, \lambda) G_{2}(s, \tau) \nu(\tau-\lambda),
\end{gathered}
$$

and

$$
\begin{aligned}
\Sigma(t)= & \frac{\hbar}{2} \int_{0}^{t} d \lambda G_{1}^{\prime}(t, \lambda) \nu(t-\lambda) \\
& -\frac{4 \hbar}{M} \int_{0}^{t} d s \int_{s}^{t} d \tau \int_{0}^{t} d \lambda \eta(t-s) G_{1}^{\prime}(t, \lambda) G_{2}(s, \tau) \nu(\tau-\lambda),
\end{aligned}
$$

where $\nu(s)$ defined as

$$
\nu(s)=\int_{0}^{+\infty} d \omega I(\omega) \operatorname{coth}\left(\frac{1}{2} \hbar \omega \beta\right) \cos (\omega s)
$$

is the noise kernel of the environment. Here a "prime" denotes taking the derivative with respect to the first variable of $G_{1}(s, \tau)$.

\section{APPENDIX B: EXPLICIT EXPRESSIONS FOR $\rho_{i j}$}

We find that the matrix $G_{i j}$ is the same for all the $\rho_{i j}$. Thus, we can write $G_{i j} \equiv G$. The matrix elements for the matrix $G$ are given by

$$
\begin{aligned}
G_{11} & =G_{22}=a_{22}+\frac{i b_{4}}{2}+\frac{i b_{8}}{2}+\frac{1}{2 \delta^{2}} \\
G_{33} & =G_{44}=a_{22}-\frac{i b_{4}}{2}-\frac{i b_{8}}{2}+\frac{1}{2 \delta^{2}} \\
G_{12} & =G_{21}=\frac{1}{2}\left(2 a_{22}+i b_{4}-i b_{8}\right), \\
G_{34} & =G_{43}=\frac{1}{2}\left(2 a_{22}-i b_{4}+i b_{8}\right), \\
G_{13} & =G_{14}=G_{23}=G_{24}=G_{31}=G_{32}=G_{41}=G_{42}=-a_{22} .
\end{aligned}
$$

Then the determinant of $G$ can be explicitly computed,

$$
\operatorname{det} G=b_{4}^{2} b_{8}^{2}+\frac{1}{16 \delta^{8}}+\frac{a_{22}}{2 \delta^{6}}+\frac{b_{4}^{2}}{2 \delta^{4}}+\frac{b_{8}^{2}}{4 \delta^{4}}+\frac{2 a_{22} b_{8}^{2}}{\delta^{2}} .
$$


Moreover, the matrix elements of the inverse matrix $G^{-1}$ are

$$
\begin{aligned}
G_{11}^{-1}=G_{22}^{-1}= & \frac{1}{\operatorname{det} G}\left(-\frac{i}{2} b_{4}^{2} b_{8}+a_{22} b_{8}^{2}\right. \\
& \left.-\frac{i}{2} b_{4} b_{8}^{2}+\frac{1}{8 \delta^{6}}+\frac{3 a_{22}}{4 \delta^{4}}-\frac{i b_{4}}{8 \delta^{4}}-\frac{i b_{8}}{8 \delta^{4}}+\frac{b_{4}^{2}}{4 \delta^{2}}-\frac{i a_{22} b_{8}}{\delta^{2}}+\frac{b_{8}^{2}}{4 \delta^{2}}\right), \\
G_{33}^{-1}=G_{44}^{-1}= & \frac{1}{\operatorname{det} G}\left(\frac{i}{2} b_{4}^{2} b_{8}+a_{22} b_{8}^{2}\right. \\
& \left.+\frac{i}{2} b_{4} b_{8}^{2}+\frac{1}{8 \delta^{6}}+\frac{3 a_{22}}{4 \delta^{4}}+\frac{i b_{4}}{8 \delta^{4}}+\frac{i b_{8}}{8 \delta^{4}}+\frac{b_{4}^{2}}{4 \delta^{2}}+\frac{i a_{22} b_{8}}{\delta^{2}}+\frac{b_{8}^{2}}{4 \delta^{2}}\right), \\
G_{12}^{-1}=G_{21}^{-1}= & \frac{1}{\operatorname{det} G}\left(\frac{i}{2} b_{4}^{2} b_{8}+a_{22} b_{8}^{2}\right. \\
& \left.-\frac{i}{2} b_{4} b_{8}^{2}-\frac{a_{22}}{4 \delta^{4}}-\frac{i b_{4}}{8 \delta^{4}}+\frac{i b_{8}}{8 \delta^{4}}-\frac{b_{4}^{2}}{4 \delta^{2}}+\frac{i a_{22} b_{8}}{\delta^{2}}+\frac{b_{8}^{2}}{4 \delta^{2}}\right), \\
G_{34}^{-1}=G_{43}^{-1}= & \frac{1}{\operatorname{det} G}\left(-\frac{i}{2} b_{4}^{2} b_{8}+a_{22} b_{8}^{2}\right. \\
& \left.+\frac{i}{2} b_{4} b_{8}^{2}-\frac{a_{22}}{4 \delta^{4}}+\frac{i b_{4}}{8 \delta^{4}}-\frac{i b_{8}}{8 \delta^{4}}-\frac{b_{4}^{2}}{4 \delta^{2}}-\frac{i a_{22} b_{8}}{\delta^{2}}+\frac{b_{8}^{2}}{4 \delta^{2}}\right), \\
G_{13}^{-1}=G_{14}^{-1} G_{23}^{-1}= & G_{24}^{-1}=G_{31}^{-1}=G_{32}^{-1}=G_{41}^{-1}=G_{42}^{-1}=\frac{1}{\operatorname{det} G}\left(a_{22} b_{8}^{2}+\frac{a_{22}}{4 \delta^{4}}\right) .
\end{aligned}
$$

For the case of $\rho_{11}$ :

$$
\begin{aligned}
\rho_{11}(t=0) & =N^{4} \exp \left[-\frac{\left(x_{10}-L_{0}\right)^{2}+\left(x_{20}-L_{0}\right)^{2}+\left(y_{10}-L_{0}\right)^{2}+\left(y_{20}-L_{0}\right)^{2}}{2 \delta^{2}}\right] \\
& \times \exp \left[i P_{0}\left(x_{10}+x_{20}-y_{10}-y_{20}\right)\right]
\end{aligned}
$$

then the matrix elements for $F$ are,

$$
\begin{aligned}
F_{11}^{1} & =i P_{0}-a_{12} x_{1}+\frac{i b_{2} x_{1}}{2}-\frac{i b_{3} x_{1}}{2}+\frac{i b_{6} x_{1}}{2}-\frac{i b_{7} x_{1}}{2}-a_{12} x_{2}+\frac{i b_{2} x_{2}}{2}-\frac{i b_{3} x_{2}}{2}-\frac{i b_{6} x_{2}}{2}+\frac{i b_{7} x_{2}}{2} \\
& +a_{12} y_{1}-\frac{i b_{2} y_{1}}{2}-\frac{i b_{3} y_{1}}{2}-\frac{i b_{6} y_{1}}{2}-\frac{i b_{7} y_{1}}{2}+a_{12} y_{2}-\frac{i b_{2} y_{2}}{2}-\frac{i b_{3} y_{2}}{2}+\frac{i b_{6} y_{2}}{2}+\frac{i b_{7} y_{2}}{2}+\frac{L_{0}}{\delta^{2}} \\
F_{11}^{2}=i P_{0} & -a_{12} x_{1}+\frac{i b_{2} x_{1}}{2}-\frac{i b_{3} x_{1}}{2}-\frac{i b_{6} x_{1}}{2}+\frac{i b_{7} x_{1}}{2}-a_{12} x_{2}+\frac{i b_{2} x_{2}}{2}-\frac{i b_{3} x_{2}}{2}+\frac{i b_{6} x_{2}}{2}-\frac{i b_{7} x_{2}}{2} \\
& +a_{12} y_{1}-\frac{i b_{2} y_{1}}{2}-\frac{i b_{3} y_{1}}{2}+\frac{i b_{6} y_{1}}{2}+\frac{i b_{7} y_{1}}{2}+a_{12} y_{2}-\frac{i b_{2} y_{2}}{2}-\frac{i b_{3} y_{2}}{2}-\frac{i b_{6} y_{2}}{2}-\frac{i b_{7} y_{2}}{2}+\frac{L_{0}}{\delta^{2}} \\
F_{11}^{3}=-i P_{0} & +a_{12} x_{1}+\frac{i b_{2} x_{1}}{2}+\frac{i b_{3} x_{1}}{2}+\frac{i b_{6} x_{1}}{2}+\frac{i b_{7} x_{1}}{2}+a_{12} x_{2}+\frac{i b_{2} x_{2}}{2}+\frac{i b_{3} x_{2}}{2}-\frac{i b_{6} x_{2}}{2}-\frac{i b_{7} x_{2}}{2} \\
& -a_{12} y_{1}-\frac{i b_{2} y_{1}}{2}+\frac{i b_{3} y_{1}}{2}-\frac{i b_{6} y_{1}}{2}+\frac{i b_{7} y_{1}}{2}-a_{12} y_{2}-\frac{i b_{2} y_{2}}{2}+\frac{i b_{3} y_{2}}{2}+\frac{i b_{6} y_{2}}{2}-\frac{i b_{7} y_{2}}{2}+\frac{L_{0}}{\delta^{2}} \\
F_{11}^{4}=-i P_{0} & +a_{12} x_{1}+\frac{i b_{2} x_{1}}{2}+\frac{i b_{3} x_{1}}{2}-\frac{i b_{6} x_{1}}{2}-\frac{i b_{7} x_{1}}{2}+a_{12} x_{2}+\frac{i b_{2} x_{2}}{2}+\frac{i b_{3} x_{2}}{2}+\frac{i b_{6} x_{2}}{2}+\frac{i b_{7} x_{2}}{2} \\
& -a_{12} y_{1}-\frac{i b_{2} y_{1}}{2}+\frac{i b_{3} y_{1}}{2}+\frac{i b_{6} y_{1}}{2}-\frac{i b_{7} y_{1}}{2}-a_{12} y_{2}-\frac{i b_{2} y_{2}}{2}+\frac{i b_{3} y_{2}}{2}-\frac{i b_{6} y_{2}}{2}+\frac{i b_{7} y_{2}}{2}+\frac{L_{0}}{\delta^{2}},
\end{aligned}
$$


where $F_{11}^{T}=\left(F_{11}^{1}, F_{11}^{2}, F_{11}^{3}, F_{11}^{4}\right)$ and

$$
\begin{aligned}
c_{11} & =-a_{11} x_{1}^{2}+\frac{i}{2} b_{1} x_{1}^{2}+\frac{i}{2} b_{5} x_{1}^{2}-2 a_{11} x_{1} x_{2}+i b_{1} x_{1} x_{2}-i b_{5} x_{1} x_{2} \\
& -a_{11} x_{2}^{2}+\frac{i}{2} b_{1} x_{2}^{2}+\frac{i}{2} b_{5} x_{2}^{2}+2 a_{11} x_{1} y_{1}+2 a_{11} x_{2} y_{1} \\
& -a_{11} y_{1}^{2}-\frac{i}{2} b_{1} y_{1}^{2}-\frac{i}{2} b_{5} y_{1}^{2}+2 a_{11} x_{1} y_{2}+i b_{1} x_{1} y_{2}+2 a_{11} x_{2} y_{2}+i b_{1} x_{2} y_{2} \\
& -2 a_{11} y_{1} y_{2}+i b_{5} y_{1} y_{2}-a_{11} y_{2}^{2}+\frac{i}{2} b_{1} y_{2}^{2}-\frac{i}{2} b_{5} y_{2}^{2}-\frac{2 L_{0}^{2}}{\delta^{2}}
\end{aligned}
$$

For the case of $\rho_{12}$ :

$$
\begin{aligned}
& \rho_{12}(t=0)=N^{4} \exp \left[-\frac{\left(x_{10}-L_{0}\right)^{2}+\left(x_{20}-L_{0}\right)^{2}+\left(y_{10}-L_{0}\right)^{2}+\left(y_{20}+L_{0}\right)^{2}}{2 \delta^{2}}\right] \\
& \times \exp \left[i P_{0}\left(x_{10}+x_{20}-y_{10}+y_{20}\right)\right] \\
& F_{12}^{1}=F_{11}^{1}, \quad F_{12}^{2}=F_{11}^{2}, \quad F_{12}^{3}=F_{11}^{3}, \quad F_{12}^{4}=F_{11}^{4}+2 i P_{0}-2 \frac{L_{0}}{\delta^{2}}, \quad c_{12}=c_{11} .
\end{aligned}
$$

For the case of $\rho_{13}$ :

$$
\begin{aligned}
\rho_{13}(t=0) & =N^{4} \exp \left[-\frac{\left(x_{10}-L_{0}\right)^{2}+\left(x_{20}-L_{0}\right)^{2}+\left(y_{10}+L_{0}\right)^{2}+\left(y_{20}-L_{0}\right)^{2}}{2 \delta^{2}}\right] \\
& \times \exp \left[i P_{0}\left(x_{10}+x_{20}+y_{10}-y_{20}\right)\right]
\end{aligned}
$$

For the case of $\rho_{14}$ :

$$
\begin{aligned}
\rho_{14}(t=0) & =N^{4} \exp \left[-\frac{\left(x_{10}-L_{0}\right)^{2}+\left(x_{20}-L_{0}\right)^{2}+\left(y_{10}+L_{0}\right)^{2}+\left(y_{20}+L_{0}\right)^{2}}{2 \delta^{2}}\right] \\
& \times \exp \left[i P_{0}\left(x_{10}+x_{20}+y_{10}+y_{20}\right)\right] \\
F_{14}^{1}=F_{11}^{1}, \quad F_{14}^{2} & =F_{11}^{2}, \quad F_{14}^{3}=F_{11}^{3}+2 i P_{0}-2 \frac{L_{0}}{\delta^{2}}, \quad F_{14}^{4}=F_{11}^{4}+2 i P_{0}-2 \frac{L_{0}}{\delta^{2}}, \quad c_{14}=c_{11}
\end{aligned}
$$

Similarly, one can work out the cases for $\rho_{2 i}, \rho_{3 i}$ and $\rho_{4 i}(i=1,2,3,4)$.

[1] M. Arndt et. al., Nature 401, 680 (1999).

[2] S. Bose, K. Jacobs and P. L. Knight, Phys. Rev. A 59, 3204 (1999).

[3] J. Friedman et. al. Nature 40643 (2000); C. H. van der Wall et. al Science 290773 (2000).

[4] A. D. Armour, M. P. Blencowe and K. C. Schwab, Phys. Rev. lett. 88, 148301 (2002).

[5] J. Eisert, M. B. Plenio, S. Bose, and J. Hartley, Phys. Rev. Lett. 93, 190402 (2004). 
[6] S. Mancini, V. Giovannetti, D. Vitali, and P. Tombesi, Phys. Rev. Lett. 88, 120401 (2002).

[7] S. Mancini, D. Vitali, and P. Tombesi, Phys. Rev. Lett. 90, 137901 (2003).

[8] W. Marshall, C. Simon, R. Penrose and D. Bouwmeester, Phys. Rev. Lett. 91, 130401 (2003).

[9] A.Bassi, E. Ippoliti and S. L. Adler, Phys. Rev. Lett. 94030401 (2005).

[10] M. Pinard et al, Europhys. Lett. 72 (5) 747 (2005).

[11] T. J. Kippenberg, H. Rokhsari, T. Carmon, A. Scherer, and K. J. Vahala, Phys. Rev. Lett. 95, 033901 (2005).

[12] A. Ferreira, A. Guerreiro, and V. Vedral, Phys. Rev. Lett. 96, 060407(2006).

[13] S. Bose, Phys. Rev. Lett. 96, 060402 (2006).

[14] E. Buks and M. P. Blencowe, quant-ph/0607106.

[15] D. W. Hallwood, K. Burnett, and J. Dunningham, quant-ph/0609077; J.A. Dunningham, K. Burnett, R. Roth, and W.D. Phillips, quant-ph/0608242.

[16] R. Feynman and F. L. Vernon, Ann. Phys. (N.Y.) 24, 118 (1963).

[17] A. O. Caldeira and A. J. Leggett, Physica A 121, 587 (1983).

[18] A sample of references is listed here: V. Hakim and V. Ambegaokar, Phys. Rev. A 32, 423 (1985); F. Haake and R. Reibold, Phys. Rev. A 32, 2462 (1985); W. G. Unruh and W. H. Zurek, Phys. Rev. D 40, 1071 (1989); H. Grabert, P. Schramm, and G. L. Ingold, Phys. Rep. 168, 115 (1988); J.J. Halliwell and A. Zoupas, Phys. Rev. D 52, 7294 (1995).

[19] B. L. Hu, J. P. Paz, and Y. Zhang, Phys. Rev. D 45, 2843 (1992); D 47, 1576 (1993).

[20] J. J. Halliwell and T. Yu, Phys. Rev. D 53, 2012 (1996).

[21] W. T. Strunz and T. Yu, Phys. Rev. A 69, 052115 (2004).

[22] C. H. Fleming, A. Roura and B. L. Hu, "Solutions to Master Equations of Quantum Brownian Motion in a General Environment with External Force" arXiv:0705.2766.

[23] C.H. Chou, B. L. Hu, and T. Yu, Physica A 387432 (2008).

[24] M. S. Kim, J. Lee, D. Ahn, and P. L. Knight, Phys. Rev. A 65, 040101(R) (2002).

[25] D. Braun, Phys. Rev. Lett. 89, 277901 (2002).

[26] M. Paternostro, W. Son, and M. S. Kim, Phys. Rev. Lett. 92, 197901 (2004).

[27] Z. Ficek and R. Tanas, Phys. Rev. A 74, 024304 (2006).

[28] R. Kubo, M. Toda, and N. Hashitsume, Statistical Physics II, (Berlin, Springer, 1991).

[29] C. W. Gardiner and P. Zoller, Quantum Noise (Berlin, Springer, 2002).

[30] This equation was originally derived for the Markovian limit. We have added the name Markov to it since we want to call the equation (30) which covers the more general cases the Fokker-Planck (FP) equation. This more general equation is sometime called the Wigner equation.

[31] C. Anastopoulos and B. L. Hu, Phys. Rev. A 62, 033821 (2000).

[32] B. L. Hu and Y. Zhang, Int. J. Mod. Phys. A 10, 4537 (1995).

[33] T. Yu and J. H. Eberly, Phys. Rev. Lett. 93, 140404 (2004); Phys. Rev. Lett. 97, 140403 (2006).

[34] P. J. Dodd and J. J. Halliwell, Phys. Rev. A 69, 052105 (2004). 
[35] J. P. Paz, S. Habib, and W. H. Zurek, Phys. Rev. D 47, 488 (1993).

[36] L. M. Duan, G. Giedke, J. I. Cirac, and P. Zoller, Phys. Rev. Lett. 84, 2722 (2000).

[37] R. Simon, Phys. Rev. Lett. 84, 2726 (2000).

[38] C. Anastopoulos, S. Shresta, and B. L. Hu, "Quantum Entanglement Under Non-Markovian Dynamics of Two Qubits Interacting With a Common Electromagnetic Field", arXiv: quant-ph/0610007

[39] L. Diosi and C. Kiefer, J. Phys. A 35, 2675 (2002).

[40] T. Yu and J. H. Eberly, Phys. Rev. B 66, 193306 (2002).

[41] K. Shiokawa and B. L. Hu, Phys. Rev. A 70, 062106 (2004).

[42] N. Cummings and B. L. Hu, "Dynamics of Atom- Field Entanglement: Towards strong coupling and non-Markovian regimes" Phys. Rev. A [arXiv:0708.2257.

[43] S. Y. Lin, C.H. Chou and B. L. Hu, in preparation.

[44] R. Landauer. IBM J Res Dev, 5, 183 (1961).

[45] C. H. Bennett, Studies in History and Philosophy of Modern Physics 34, 501 (2003).

[46] C. M. Caves, Phys Rev E, 47, 4010 (1993).

[47] J. Bub, Studies in the History and Philosophy of Modern Physics, 32, 569 ( 2001).

[48] O. J. E. Maroney, "Generalising Landauer's Principle" arXiv:quant-ph/0702094. "The physical basis of the Gibbs-von Neumann entropy" arXiv:quant-ph/0701127. "Information and Entropy in Quantum Theory" PhD thesis, Birkbeck College, University of London, 2002. arXiv:quant-ph/0411172.

[49] C. Hoerhammer, H. Buettner, "Information and entropy in quantum Brownian motion: Thermodynamic entropy versus von Neumann entropy" arXiv:0710.1716.

[50] C. Hoerhammer, H. Buettner, J. Phys. A 38, 7325 (2005).

[51] C. H. Fleming, A. Roura and B. L. Hu, "Solutions to Master Equations of Quantum Brownian Motion in a General Environment with External Force" arXiv:0705.2766.

[52] D. Vitali et al, Phys. Rev. Lett. 98, 030405 (2007).

[53] C. Genes, D. Vitali, P. Tombesi, S. Gigan, M. Aspelmeyer. "Ground-state cooling of a micromechanical oscillator: generalized framework for cold damping and cavity-assisted cooling schemes" [Xiv:0705.1728].

[54] H. Mueller-Ebhardt, H. Rehbein, R. Schnabel, K. Danzmann, Y. Chen, "Entanglement of macroscopic test masses and the Standard Quantum Limit in laser interferometry" arXiv:quant-ph/0702258.

[55] T. Yu, C. Fleming and B. L. Hu, "Master equation for Macroscopic Quantum Phenomena in MirrorPhoton Systems" in preparation.

[56] C. Galley, C. H. Chou and B. L. Hu, "Quantum Superposition of Two Mirrors mediated by photons: Modeling via two harmonic oscillators in a common bath" in preparation. 